This is a preprint of an article published in Developmental Cell. The final authenticated version is available online at: https://doi.org/10.1016/j.devcel.2021.07.005

\title{
A reflux-and-growth mechanism explains oscillatory patterning of lateral root branching sites
}

Thea van den Berg ${ }^{1}$ [1], Kavya Yalamanchili ${ }^{1}$ [2], Hugues de Gernier [3], Joana Santos Teixeira[1], Tom Beeckman² [3], Ben Scheres ${ }^{2}$ [2,4], Viola Willemsen² [2] and Kirsten ten Tusscher* [1]

[1] Utrecht University

[2] Wageningen University

[3] University Ghent

[4] Rijk Zwaan Breeding B.V.

1,2These authors contributed equally

*Lead author, correspondence: k.h.w.j.tentusscher@uu.nl

\begin{abstract}
Modular, repetitive structures are a key component of complex multi-cellular body plans across the tree of life. Typically, these structures are prepatterned by temporal oscillations in gene expression or signaling. While for vertebrate somitogenesis and arthropod segmentation a clock-and-wavefront mechanism was identified and plant leaf phyllotaxis arises from a Turing-type patterning, the mechanism underlying lateral root patterning has remained elusive.

To resolve this enigma we combined computational modelling with in planta experiments. Intriguingly, auxin oscillations automatically emerge in our model from the interplay between a reflux loop generated auxin loading zone, and stem cell driven growth dynamics generating periodic cell size variations. In contrast to clock-and-wavefront and Turing patterning, the uncovered mechanism predicts both frequency and spacing of lateral root forming sites to positively correlate with root meristem growth. We validate this prediction experimentally. Combined, our model and experimental results support that underlying lateral root priming is a novel periodic patterning mechanism.
\end{abstract}

\section{Introduction}

In multi-cellular organisms, developmental processes are not only responsible for generating a multitude of cell types from genetically identical cells, but also for the spatial organization of cell types, tissues and organs into functional body plans. In many multi-cellular organisms, at least part of the body plan is of modular, repetitive nature. Well-known examples are the segments of annelids and arthropods (Davis and Patel, 1999), the somites (Conlon et al., 1995; Dubrulle and Pourquié, 2002; Palmeirim et al., 1997), and appendages of vertebrates (Dolle et al., 1989; McGinnis and Krumlauf, 1992; Saunders, 1948) as well as the phytomers of plants (Reinhardt et al., 2003; Smith et al., 2006), trichome patterning on plant leaves (Ishida et al., 2008; John C. Larkin et al., 1999) and placement of hair cells on roots (Galway et al., 1994; Ishida et al., 2008). Plant root system architecture is also repetitive, but on top of this, displays high plasticity, ensuring access to water and nutrients under a wide range of different conditions (Eshel and Beeckman, 2013; Rogers and Benfey, 2015). As a consequence, under natural conditions the regular nature of the root system architecture may become obscured (Gruber et al., 2013). In the model plant Arabidopsis thaliana it has been shown that the earliest step in the formation of new lateral roots, essential for the formation of a branched root architecture, is highly regular (De Smet et al., 2007; Moreno-Risueno et al., 2010). Lateral root (LR) formation starts with the priming of subsets of pericycle cells to gain competence for the future formation of LRs (De Smet et al., 2007; MorenoRisueno et al., 2010). Priming is characterized by temporal oscillations in auxin signaling and gene expression in the root transition zone with an as yet unknown primary cause. Primed sites will through growth become transformed into a spatially repetitive pattern of competent sites for LR 
formation (De Smet et al., 2007; Moreno-Risueno et al., 2010; Xuan et al., 2015; Xuan et al., 2016).

Mathematical modelling has played a key role in unravelling the mechanisms underlying periodic developmental patterning processes. In 1952 Turing demonstrated that interactions between a slowly diffusing activator and a more rapidly diffusing inhibitor substance could give rise to regular periodic patterning (Turing, 1952). In case of polar tissue outgrowth such Turing type patterning mechanisms give rise to the periodic production of new pattern elements. While originally proposed for phyllotaxis (Saunders, 1992), and for a long time difficult to substantiate experimentally, Turing type patterns are now generally accepted to underly vertebrate appendage (Newman and Frisch, 1979) and digit patterning (Raspopovic et al., 2014), and hair (Plikus et al., 2008; Sick et al., 2006), feather (Jiang et al., 1999; Shyer et al., 2017) and skin pigmentation patterning (Yamaguchi et al., 2007). Additionally, a Turing-like substrate depletion mechanism in which up-the-gradient polarization of auxin exporting PIN proteins locally enhances, and at a longer range depletes, auxin levels has been shown to underly phyllotaxis (Jonsson et al., 2006; Reinhardt et al., 2003; Sick et al., 2006). Of similar significance is the clock-and-wavefront mechanism first proposed by Cooke and Zeeman in 1976 (Cooke and Zeeman, 1976). This model proposes that underlying somitogenesis is a cell-autonomous oscillatory clock combined with a wavefront dictating when and where these temporal oscillations become translated into a periodic pattern. The clock-andwavefront model has received substantial experimental support, identifying the clock as a set of genetically encoded negative feedbacks resulting in gene expression oscillations (Bessho et al., 2003; Dequeant et al., 2006; Panganiban et al., 1997; Sarrazin et al., 2012). Based on the observation of periodic variations in gene expression coinciding with variations in auxin signaling, Moreno-Risueno (2010) proposed that a clock-and-wavefront mechanism analogous to that observed in vertebrate somitogenesis underlies periodic priming (Moreno-Risueno et al., 2010). As an alternative possibility a Turing-type mechanism, similar to that used in vertebrate appendage patterning and phyllotaxis has been proposed (Laskowski and Ten Tusscher, 2017). Thus far, for neither mechanism substantial proof has been provided.

So far, no single loss-of-function mutations have been identified that completely abolish LR formation at or preceding the founder cell stage, while mutations blocking LR formation at later stages have been identified (DiDonato et al., 2004). Interestingly, a strong repression of LR formation occurs in the dark, known to affect sugar transport and consequently root growth dynamics (Jensen et al., 1998). Additionally, studies have demonstrated an important role for the synthesis of the auxin precursor indole-3-butyric acid (IBA) in the lateral root cap (LRC) in determining the amplitude of priming oscillations (Strader and Bartel, 2011; Xuan et al., 2015) and reported a reduced production of LRs for mutations in auxin transporting proteins such as PIN2 (Xuan et al., 2016), LAX3 (Lewis et al., 2011; Swarup et al., 2008) and AUX1 (De Smet et al., 2007; Lewis et al., 2011; Xuan et al., 2015). Auxin perception in the vasculature was furthermore shown to be critical for LR formation (De Smet et al., 2007). Finally, recent studies reported a strong spatio-temporal coincidence of repetitive LRC apoptosis and priming events (Xuan et al., 2016) as well as a key role for growth related cell-wall remodeling (Wachsman et al., 2020). Together, these studies indicate the importance of auxin production, transport and perception while also hinting at a key role for growth dynamics.

Here we hypothesized that the interplay between the auxin reflux loop and growth dynamics gives rise to regular auxin oscillations. To investigate this hypothesis we combined computational modelling and in planta experiments. Excitingly, incorporating auxin transport and growth dynamics into a novel multi-scale realistic root model automatically led to the emergence of repetitive auxin peaks. Moreover, these auxin oscillations originated in the elongation zone (EZ) protoxylem and were subsequently transmitted to the pericycle, consistent with experimental observations (De Smet et al., 2007). Specifically, we found that root tip anatomy and reflux loop properties result in an auxin loading zone at the start of the $E Z$, with preferential loading in large narrow vasculature cells. Additionally, we showed how root growth dynamics in the meristem, by causing alternations in the sizes of cells arriving at the transition zone (TZ), causes substantial variation in auxin loading potential, resulting in auxin level oscillations. In contrast to a Turing mechanism or a clock-and-wavefront mechanism the 'reflux-and-growth' mechanism discovered 
here predicts that both the frequency and spatial spacing of priming events depend on meristem cell production and hence tissue growth rate. Our in planta experiments confirmed these model predictions. Combined, our findings support that a hitherto undescribed mechanism for periodic pattern formation underlies LR priming. Our in planta experiments uncovered similar correlations for actual formed LRs as for the priming sites, indicating the importance of early priming events for actual root branching patterns.

\section{Results}

\section{Priming emerges from combined root auxin transport and growth dynamics}

To investigate the role of root tip auxin reflux and growth dynamics in the generation of auxin oscillations, we developed a novel computational model, incorporating an anatomically realistic root topology and detailed auxin dynamics. The model incorporates cell type and zone specific patterns of the auxin exporting PIN membrane proteins as well as the auxin importing AUX/LAX membrane proteins, as well as passive cellular auxin import, auxin production and degradation, and a shoot-derived influx of auxin. Additionally the model incorporates realistic root growth dynamics consisting of slow stem cell (SC) divisions near the quiescent centre (QC), subsequent clonal expansion of more rapidly dividing transit amplifying (TA) cells (Bizet et al., 2015; Rahni and Birnbaum, 2019) as well as expansion and differentiation dynamics (Fig 1A). As a consequence, after originating from a SC division, individual cells sequentially transition these distinct zones as younger cells formed rootward of them push them away from the SC niche. Additionally, individual cells undergo dynamic cell size changes. While growing in between divisions cells gradually increase in size, halving their size upon division, and when undergoing expansion they undergo a much more rapid size increase that ends as cells start their terminal differentiation.

Since we are focusing on LR priming, in which protoxylem and xylem pole pericycle play critical roles, our two-dimensional model represents a longitudinal cross-section through the protoxylem poles. To include critical aspects of in planta three dimensional auxin fluxes (el-Showk et al., 2015) in addition to the predominant basally oriented active auxin transport, we also incorporated outward oriented, protoxylem directed PIN transport in the vasculature (Fig. S1A).

Surprisingly, regular temporal variations in auxin levels automatically emerged in this model (Fig. $1 \mathrm{~B}$, Video S1). Even more noteworthy, these oscillations predominantly occur in the vasculature (Fig. 1B) and pericycle (Fig. S1B) of the TZ and early EZ, precisely where priming is experimentally found to occur (De Smet et al., 2007). Indeed, as is clear from the root tip auxin patterns, highest auxin levels occur in the vascular tissues (Fig. S1B).

\section{Auxin availability can shape oscillation amplitude}

Previous research indicated the importance of auxin production for priming amplitude (De Rybel et al., 2012; Dubrovsky et al., 2008; Strader and Bartel, 2011; Xuan et al., 2015). For example, LRC specific production of the auxin precursor IBA was found to significantly enhance the amplitude of priming oscillations (Strader and Bartel, 2011; Xuan et al., 2015). To investigate the consistency of the priming mechanism occurring in our model with these observations, simulations with reduced LRC auxin production were performed. Consistent with experimental results, oscillation amplitude was reduced (Fig. 1C) while frequency was unaffected (Fig. S1C). Importantly, similar reductions in stem cell region auxin production or shoot auxin influx resulted in similar reductions in oscillation amplitude (Fig. 1C, S1C), indicating that overall auxin availability rather than its location of origin is relevant for oscillation amplitude. Furthermore, when normalizing auxin levels against total root tip auxin content (Fig. 1D), we find that amplitude changes can be fully ascribed to changes in overall auxin availability.

\section{Root tip reflux loop can define a TZ/EZ auxin loading domain}

While shoot influx and local auxin production control root tip auxin availability, spatio-temporal auxin patterning strongly depends on auxin transport (Band et al., 2014; Grieneisen et al., 2007). Previous experimental research has reported reduced LR production for mutations in PIN2 (Xuan et al., 2016), LAX3 (Lewis et al., 2011; Swarup et al., 2008) and AUX1 (De Smet et al., 2007; Lewis et al., 2011; Xuan et al., 2015). To assess the importance of the root tip auxin reflux loop for the auxin oscillations uncovered in our novel model, simulations with modified PIN and AUX/LAX expression patterns were performed. First, we simulated auxin dynamics in the presence of only 
vascular localized, rootward oriented PINs, representing a pin2 null mutant in absence of other PINs taking over upward transport. This resulted in a nearly complete abolishment of auxin oscillations even when normalized against the significantly reduced total root auxin content (Fig. 1E, S1D). Next, we investigated auxin patterning when PIN2 levels were reduced by $2 / 3$, which caused (normalized) oscillation amplitude to decrease with $33 \%$ (Fig. 1E, S1D). If instead we maintain PIN2 levels yet remove lateral inward oriented PIN2 in the epidermis, oscillations decrease in amplitude and shift shootward (Fig. 1E, S1D). Finally, we simulated an aux1 mutant, resulting in a $67 \%$ decrease of (normalized) auxin oscillation amplitude (Fig. 1E, S1D), consistent with earlier observations that absence of AUX1 expression in the LRC strongly reduces oscillation amplitude (De Smet et al., 2007; Xuan et al., 2016). Combined, these results suggest that PIN2 and AUX1 mediated upward transport through the LRC and epidermis, and subsequent inward transport towards the vasculature are essential for auxin oscillations in the vasculature of the TZ and EZ. Noteworthy, the previously proposed LRC apoptosis driven priming mechanism relies on a similar upward and inward auxin transport route (Xuan et al., 2016).

\section{Growth of vasculature and pericycle is a key component of the auxin oscillation model}

Previous experimental results demonstrated a strong correlation between periodic LRC apoptosis and oscillatory LR priming (Xuan et al., 2016). To test the causal nature of this correlation in our model, simulations mimicking defects in LRC growth dynamics were performed. Outcomes show that auxin oscillations persist albeit at reduced amplitude both in a simulated sombrero $(\mathrm{smb})$ mutant (Fig. 2A, S2A), as well as in complete absence of LRC growth dynamics (Fig. 2A, S2A). These results suggest that other coordinated growth processes may be responsible for the correlation between LRC apoptosis and auxin oscillations but not LRC shedding itself. To further explore this, we systematically simulated the consequences of growth dynamics in a subset of tissues while not applying growth elsewhere. Growth of vasculature and pericycle tissue was found necessary and sufficient for auxin oscillations, while growth of other tissue layers merely contributes to oscillation amplitude (Fig. 2B, S2B). To further support our finding that only growth of the to be primed tissues is required we performed simulation in a minimal 1D model with a single row of growing cells with similar SC niche driven growth dynamics as used in the full model. The results show that mild auxin oscillations arise that are augmented when adding an auxin loading zone (Fig. S2C).

\section{Growth induced cell size increases can drive auxin loading into vasculature cells}

Auxin availability and a functional reflux loop explain how auxin becomes transported upward to the TZ/early EZ, but do not yet explain why vascular and pericycle growth are essential for this process, nor why auxin oscillations predominantly occur in these tissues. Indeed, while the lateral, outward oriented vascular PIN pattern explains the within-vasculature preference for the outermost, protoxylem cell file, it does not explain the preferential loading of auxin in the vasculature per se. Particularly since this loading pattern implies that the auxin transported in the reflux loop passes from the $\mathrm{LRC}$, through epidermis and ground tissue, to the vasculature, and, albeit with somewhat different amplitude and location, still occurs in absence of inward oriented PIN2 in the epidermis, root cap and cortex.

We hypothesized that cell growth causes auxin increase through an enhanced auxin influx/efflux ratio: While the typically apolar active and passive auxin import increases with membrane surface area and hence cell size, the predominant polar localization of PIN exporters on non-growing rootward (PIN1) and shootward (PIN2) membranes causes a relative decrease in auxin efflux with increasing cell sizes. Notably, these effects will be more prominent for narrow vasculature cells that undergo the largest increase in surface to volume ratio. Decreasing the width of only vascular tissue in our model further enhances auxin oscillation amplitude(Fig. 2C), corroborating this hypothesis. Simulations normalizing either active AUX1 mediated auxin influx, passive membranebased auxin influx or active PIN mediated auxin efflux with cell height indicate that auxin oscillations are predominantly driven by a size-dependent increase in passive auxin uptake (Fig. 2D).

\section{An explanation for progress of priming from vasculature to pericycle}


In experiments, priming is initially observed in protoxylem cells, with the signal subsequently being passed on, and in case of successful priming, maintained in pericycle cells (De Smet et al., 2007). In our default settings, priming occurs simultaneously in the protoxylem and neighboring pericycle, with the two tissues experiencing only mildly different maximum auxin levels (Fig. S1B). However, in planta, protoxylem, protophloem and metaphloem cells stop dividing closest to the root tip, while neighboring pericycle cells do so considerably further shootward (Lavrekha et al., 2017). These different dynamics will result in large protoxylem cells next to smaller pericycle cells at the start of the TZ. After incorporating these characteristics into our model we observed auxin elevations to first occur in the vasculature, and subsequently being passed on to abutting pericycle cells (Fig. $2 \mathrm{E}, 2 \mathrm{~F}$ ), in line with experimental data (De Smet et al., 2007). Notably, the differential onset of cell expansion enhances vascular oscillation amplitude while reducing that of the pericycle and allows for a partial maintenance of the priming signal (Fig. 2E, 2F). Thus, our model naturally produces the correct location and order of events in LR priming, as an emergent property of incorporating increasingly realistic root growth dynamics.

\section{Priming occurs in large cells followed by smaller cells}

Our results thus far indicate that priming arises from the preferential loading of auxin in large, rapidly expanding vascular cells, but do not yet explain what causes its periodic nature. To decipher the mechanism underlying the periodic nature of the observed auxin oscillations, we first compared kymographs depicting intracellular auxin levels with those showing cell size (Fig. 3A). Additionally, we correlated cell size and auxin loading dynamics (Fig. 3B). In both kymographs and plot we observe a strong correlation between cell size upon entering the $E Z$ and maximum attained auxin levels, confirming our earlier results on the importance of cell size for auxin uptake. In addition to cell height, we observed a weaker but significant correlation for cellular auxin levels with $E Z$ residence time. This can be understood from the fact that EZ residence time affects loading time as well as the amount of cell size increase occurring inside this loading zone (Supp. 1).

Next, we focused on the periodicity in auxin loading and its predominant relation to cell size. In the kymographs we see that maximum auxin loading occurs in the latest, largest arriving cells of a group of incrementally larger and more auxin loading cells, with this group being terminated by newer, smaller cells arriving (Fig. 3A). These smaller cells, which by growing slower cause a slower displacement of shootward cells, enhance the EZ residence time of larger cells, thereby further boosting their size dependent auxin loading (Supp 1, Fig 3B). As maximum auxin loading thus occurs on the boundary of large and small cells arriving at the EZ, we can effectively trace the temporal dynamics of priming by focusing on the periodic occurrence of directly abutting largesmall cell pairs (Fig. 3B: $>1$ ratio for cell height/cell height below cell).

The sequential increase in cell size terminating with a small cell starting of a new sequence observed in our model was previously observed for cortical cells in a detailed root tip tracking study(von Wangenheim et al., 2017) . Unfortunately, direct validation of the priming mechanism uncovered here by concurrently measuring detailed cell size and auxin dynamics in planta is impossible with current techniques, particularly for the narrow shaped and internally localized vasculature. Similarly, validation through tracing back LR forming sites to earlier, only transiently present, cell size differences or spatio-temporally targeted interference with the formation of largesmall cell pairs, or their cell-size dependent auxin loading is technically unfeasible. Therefore, we decided to further unravel how root growth dynamics determines priming characteristics in order to arrive at a series of experimentally testable predictions on the relation between root growth and priming features.

\section{Priming arises from abutting clones in a defined spatio-temporal window}

First, simulations were performed varying different components of overall root growth: TA cellular division rate, meristem size (i.e. number of dividing cells), and cellular elongation rate. Results show that both increases in TA division rate (for a given meristem size) and meristem size (for a given TA division rate) enhance priming frequency (Fig. 4A, 4B, S4A, S4B, Video S4a, Video S4b). In contrast, increases in elongation rate enhance priming amplitude (Fig. 4C, Video S4c) but not frequency (Fig. 4D). Thus, overall meristem cell production, determined by both division frequency and meristem size, dictates priming frequency. 
Next we set out to unravel how meristem cell production determines priming dynamics, i.e. the periodic formation of large-small cell pairs. For this we need to consider that plant root growth is directionally organized, with rootward oriented, slowly dividing stem cells generating transit amplifying (TA) daughter cells that enter the meristem (Bizet et al., 2015; Lavrekha et al., 2017; Rahni and Birnbaum, 2019). These TA cells undergo multiple rounds of rapid, approximately synchronized divisions, forming an expanding clone of sibling cells (Rahni and Birnbaum, 2019; von Wangenheim et al., 2017). Due to the formation of newer, out-of-phase, clones, older clones move shootward relative to the QC, out of the meristem.

Formation of a large-small cell pair requires that for two abutting, out-of-phase clones the rootward clone divides once more after the last division of the top clone. The chances at any given meristem location for two such abutting clones to occur depend on clone density. As a measure for the number of clones fitting in the meristem, we take the number of divisions a cell can undergo before leaving the meristem, given by $\log _{2}$ (meristem size) (Beemster and Baskin, 1998) (Fig. 5A). Thus, clone density scales with $\log _{2}$ (meristem size)/ meristem size, indicating that as MZ size increases, clone density decreases and clone size increases (Fig. 5B).

Additionally, to produce a large-small cell pair at the TZ, the final division in the top clone has to occur sufficiently early for it to have time to grow large, yet not so early that it undergoes another division and becomes small again. Thus this final division should occur in a limited spatial window, defined by the position cells occupy when at a distance of 1-(1- $\alpha)$ cell cycle from the TZ, with $\alpha$ sufficiently small. Indeed, tracing back primed cells to the position of their final divisions reveals a restricted spatial window, with a value for $\alpha$ of 0.25 (Fig. 5C). Larger meristems result in a faster cumulative displacement of cells, translating this temporal window into a spatial window (in number of cells) that increases linearly with meristem size (Fig. 5D) (see Supp. 2).

Given that per meristem position the chances for two out-of-phase clones scales with $\log _{2}$ (meristem size)/meristem size, and priming requires the top clone to divide within a spatial window which size scales with meristem size, priming frequency should scale with $\log _{2}$ (meristem size), consistent with our simulations showing a sublinear increase with meristem size (Fig. 4B, 5D). However, this only takes into account the chance for two abutting clones to occur at a position at which this could potentially lead to formation of a large-small cell pair. For this to actually occur, the topmost clone should divide at this position, which occurrence scales with TA division frequency (Fig. 4A, 5E). Overall priming frequency thus depends on the product of division rate with $\log _{2}$ (meristem size).

By definition, the spacing between primed sites (PS) is equal to the number of cells passing by in between priming events, and hence equals cell production divided by priming frequency. A sublinear dependence of priming frequency on meristem size (Fig. 4B), implies that as meristem size (and hence production) increases, PS spacing should increase as well. Indeed, in our simulations we see a strong positive correlation between PS spacing and meristem size (Fig. 6A). On the contrary, since increases in division frequency almost fully translate to increases in priming rate (Fig. 4A) (Supp 1.), we expect no significant correlation between PS spacing and division rate, Indeed simulations confirm that this correlation was not significant (Fig. 6B).

Summarizing, our model predicts a positive correlation between priming frequency and meristem size as well as division rate (Fig. 4A, 4B), and between PS spacing and meristem size (Fig. 6A).To more compactly represent our findings we translate these predictions into a positive correlation between priming frequency and spacing with cell production (product of meristem size and cell division rate) (Fig. 6C, 6D). Additional advantage of this compression is that we can now display all data points combined, rather than using only a subset of datapoints with similar meristem size when plotting priming as a function of division rate, or vice versa (i.e. Fig. 4A, 4B).

Validation of the reflux-and-growth priming mechanism. Our reflux-and-growth model thus predicts a positive relationship between both priming frequency and spacing and cell production (Fig $6 \mathrm{E}$ and $6 \mathrm{~F}$, green lines). As a next validation step, we compared these predictions against those of 
alternative models, and investigate which model predictions are supported best by the experimental data. A first alternative model is the clock-and-wavefront model (Moreno-Risueno et al., 2010), generally accepted to underlie the segmentation of the vertebrate body axis into somites(Baker et al., 2006; Conlon et al., 1995; Dubrulle et al., 2001; Jiang et al., 2000; Palmeirim et al., 1997). Following to the clock-and-wavefront paradigm, oscillation frequency (and thus presumably priming frequency and LR production rate) depends on the frequency of the cellautonomous clock, generated by a delayed negative feedback motif, and would be independent of meristematic cell production (Fig. 6E, black line). In contrast, wavelength (i.e. PS spacing) depends on the amount of growth in between clock periods and would hence scale with production (Fig. 6F, black line)

Another theoretically possible mechanism that has been proposed for LR priming is the Turing mechanism (Laskowski and Ten Tusscher, 2017; Turing, 1952). In a polarly growing tissue such as the root, the continuous increase of tissue size would result in the periodic production of new peaks each time tissue size has increased with the wavelength of the Turing pattern. This causes LR production rate to linearly depend on growth rate and hence cell production (Fig. 6E, red line). The wavelength itself depends on the interaction and transport parameters together giving rise to the Turing pattern, and are independent of cell production (Fig. 6F, red line).

To investigate whether in planta priming dynamics support the reflux-and-growth priming mechanism uncovered here we set up experiments aimed at inducing a range of different meristem cell production rates and resulting priming dynamics. To this end, we compared plants grown under control conditions with plants treated with the growth hormones, gibberellic acid (GA), and two different levels of brassinosteroids (BR), to induce variation in relevant growth parameters and thereby priming (Gonzalez-Garcia et al., 2011; Ubeda-Tomas et al., 2008). Importantly, while these hormonal treatments through cross-talk may interfere with auxin levels and/or signaling, both our model and experimental data (Perianez-Rodriguez et al., 2021; Xuan et al., 2016) indicate that this only directly affects oscillation amplitude, not frequency. This enables us to focus on the direct and indirect effects of these hormone treatments on meristem growth dynamics and its relation with priming dynamics.

To achieve this we designed an experimental set-up following priming zone auxin oscillations in time through recording activity of a DR5::LUC transgene (Moreno-Risueno et al., 2010), while growth parameters were tracked in parallel. Overall, sizes of actively dividing meristems range from $\sim 12$ to $\sim 48$ cortical cells ( $\sim$ fold range) (Fig. 6G, S6A), cell production rates from 1.23 to 3.2 ( 2.5 fold range) (Fig. 6G, S6B), priming frequency from 0.2 to 0.87 events per hour ( 4.5 fold range) (Fig. $6 \mathrm{H}, \mathrm{S} 6 \mathrm{C}$ ) and priming site spacing from 1 to 8 cells ( $~ 8$ fold range) (Fig. $6 \mathrm{H}, \mathrm{S} 6 \mathrm{D}$ ). Thus, significant variations in growth parameters as well as priming characteristics were successfully generated using the selected treatments.

Our experimental perturbation data show a significant positive correlation between both priming frequency and PS spacing with cell production (Fig. 7A, 7B, S7A, S7B), as well as similar relations between frequency and spacing with meristem size and division frequency (Fig. S7C, S7D, S7E, S7F ). This enables us to falsify both Turing based and clock-and-wave front driven priming mechanisms and support the newly discovered reflux-and-growth priming mechanism.

\section{Distinct vascular division dynamics significantly contribute to priming frequency}

Interestingly, despite our model simulations covering active meristem sizes and division rates over a range similar to those measured experimentally, priming frequencies and PS spacing distances observed in our model differ approximately $\sim 4$ fold from those experimentally measured (compare Fig. 6C and 6D with, 7A and 7B). Given that the model does correctly simulate correlations between priming frequency, PS spacing and meristem cell production, we reasoned that while the priming mechanism found in our model is correct, some quantitative aspect must differ from the in planta situation.

Additionally to the aforementioned early onset of differentiation in the protoxylem relative to the pericycle (Lavrekha et al., 2017), two recent studies measuring Arabidopsis root cell division 
dynamics found that transit amplifying division frequencies in stele cells are approximately 1.5 times higher as compared to cortical cells (Lavrekha et al., 2017; Rahni and Birnbaum, 2019). This implies that our measured cortical cell production and division rates significantly underestimate the rate of vascular divisions driving priming dynamics. Additionally, one of these studies reported that while cellular division rates were higher, the frequency of division events within the stele was lower and cell length larger, indicating fewer, larger cells (Lavrekha et al., 2017). Incorporating these three vasculature specific features into our model resulted in a substantial increase in priming frequency and decrease in PS spacing (Fig.7C, 7D, red versus green data), maintaining the previously found relationships while significantly improving the quantitative agreement between model and experimental data (Fig. S7G and S7H).

\section{Actual LR frequency and PS spacing scale in similar manner with cell production}

For plant fitness, it is not the frequency and spacing of priming events, but the actual formation and spacing of LRs that is relevant. A major question thus is to which extent our model is capable of explaining LR patterning. To establish this, an additional series of experiments was performed, using similar perturbations as before, but now counting the numbers of and distances between LRs as well as LR primordia, using the early-expressed LR specific PLT3 reporter (Du and Scheres, 2017). Figure 7E and 7F shows that a similar positive correlation between LR formation frequency and LR spacing and cell production was found as for PS frequency and spacing. Thus, with our unravelling of the mechanisms underlying LR priming, also meaningful predictions for actual LR patterning can be made.

\section{Discussion}

At first glance, the root system of a plant does not necessarily appear highly regular (Gruber et al., 2013), yet the initial step of LR formation, LR priming, has been shown to have a repetitive nature. During LR priming, periodic oscillations in the plant hormone auxin and its downstream effects prepattern subsets of pericycle cells to become competent for future LR formation (De Smet et al., 2007; Moreno-Risueno et al., 2010; Xuan et al., 2015; Xuan et al., 2016). Later stages of lateral root formation have successfully been unraveled. As an example the transition from 2 primed protoxylem sites to a single sided stable founder cell has been shown to arise from a competition for auxin (signaling) (el-Showk et al., 2015) that can be biased by environmental factors such as water availability promoting auxin signaling intensity(Orosa-Puente et al., 2018). Thus far, the mechanistic basis of lateral root priming has remained unclear.

In this study we aimed to uncover the mechanism underlying LR priming. Inspired by the reported importance of auxin transport as well as the strong correlation with root cap apoptosis, root growth and cell wall remodeling (Jensen et al., 1998; Wachsman et al., 2020; Xuan et al., 2016) we hypothesized that priming arises from an interplay between root tip auxin transport and growth dynamics. To test this idea, we developed a novel multi-scale root growth model enabling us to simultaneously track auxin and auxin signaling dynamics at the single cell, tissue and whole root organ level, while independently varying different aspects of auxin and growth dynamics. Analyzing our novel growth model, we uncovered that the root tip auxin reflux loop creates an auxin loading domain at the start of the EZ. Additionally, we elucidated how their narrow shape and early onset of elongation provides vasculature cells with the highest auxin loading potential, causing preferential auxin accumulation in these cells. Next we showed how priming occurs in the final, largest cell of a series of increasingly large vasculature cells, and is followed by a significantly smaller cell. We then went on to demonstrate how the typical root tip growth dynamics results in the periodic production of large-small cell pairs. Specifically, we elucidate how stem cell driven growth dynamics results in the sequential production of out-of-phase clones of sibling cells, and hence out-of-phase dividing neighboring cells. Combined we thus uncovered a novel reflux-andgrowth mechanism for periodic LR priming. To link this model to experiments, we derived predictions stating that both cell division rate and meristem size, and hence meristem cell production rate determines priming frequency as well as PS spacing.

To validate these model predictions, we measured spatio-temporal dynamics of priming site and LR formation using perturbations that allowed us to extract information on the influence of 
individual model parameters by deconvolution of effects. In agreement with our model predictions but not with those of competing models, we observed a significant positive correlation of both priming frequency and spacing with meristem cell production rate. Our results thus present a novel framework explaining the periodic nature of LR priming in dicot plant roots that highlights the role of cell size differences in auxin-based patterning. As such it bears resemblance to an earlier study demonstrating how lateral root bending, through enlarging cells at the outer curve of the bend, could induce lateral root formation (Laskowski et al., 2008).

As a further support of the mechanism we propose here, agreement between model outcomes and experimental data further increased when incorporating additional biological detail into our model. When accounting for the differences in distance relative to the QC from where cells stop dividing (Lavrekha et al., 2017), our model automatically reproduces the start of priming in the protoxylem file (De Smet et al., 2007), and the subsequent transmission of the signal to the pericycle, where founder cell formation occurs (Dubrovsky et al., 2006). On a similar note, incorporating the experimentally observed larger size and higher division frequency of vascular cells (Lavrekha et al., 2017; Rahni and Birnbaum, 2019) significantly improved quantitative agreement between model and data. Additionally, the predicted periodic pattern of gradually increasing sizes of cells arriving at the transition zone with the final largest cell followed by the first smallest cell of a new sequence has been measured for cortical cells in a detailed root tip tracking study)(von Wangenheim et al., 2017).

Compared to prebranch site spacing actual root system architecture is often considerably less regular (Gruber et al., 2013). Stochasticity (Laskowski, 2013), root curvature (Kircher and Schopfer, 2016; Richter et al., 2009), environmental conditions (Bao et al., 2014; Gruber et al., 2013), and competition between neighboring primed sites (Bielach et al., 2012; Hofhuis et al., 2013; Toyokura et al., 2019) all mold prebranch site patterns into actual root system architecture. Indeed, we observed $\sim 4$ fold lower LR numbers and $\sim 4$ fold increase in LR spacing as compared to priming site numbers and spacing (Fig. 7A, B and Fig. 7E,F). Although differences may partly arise from data being obtained in separate experiments under slightly different conditions, the size of the difference suggests many priming events do not result in LR formation. While earlier studies reported a near $100 \%$ translation from priming events to LR formation (Xuan et al., 2015; Xuan et al., 2016), much lower success rates were reported in experiments using a similar intermittent light regime and no additional sucrose as we applied here (Kircher and Schopfer, 2018). Combined, this underlines the extensive reserve potential and plasticity in root architecture development under more natural growth conditions. Nevertheless, our in planta experiments demonstrate that for actual formed LRs a similar dependence of frequency and spacing on meristem growth rate exists as was found for prebranch sites. Thus, at least for idealized experimental conditions, qualitative spatio-temporal characteristics of priming and prebranch site patterning translate into those for LRs.

In addition to the predictions of our model being borne out by our experimental outcomes, the priming mechanism uncovered here agrees with a large range of experimental data. We demonstrated how reduction of LRC auxin production (Xuan et al., 2015), and mutations in auxin transporters (De Smet et al., 2007; Lewis et al., 2011; Swarup et al., 2008; Xuan et al., 2015; Xuan et al., 2016) significantly reduce oscillation amplitude, explaining the reduced observed prebranch site numbers from a decreased chance for priming to lead to prebranch site formation. Also, the predicted importance of cell elongation for oscillation amplitude (Fig. 4C, 4D) is supported by recent experimental findings reporting reduced LR formation for roots with shorter elongated cells (Alarcon et al., 2019), or reduced potential for cell wall modification (Wachsman et al., 2020) . Additionally, the central role of growth uncovered here explains why in absence of light and hence growth, no LR formation takes place (Jensen et al., 1998). The observed relationship between LR formation and cell production is furthermore supported by studies varying nutrient levels, where a decrease in main root size (and hence cell production) translated to reduced LR numbers and a higher LR density (Gruber et al., 2013; Pérez-Torres et al., 2008). Our findings furthermore suggest that the previously found correlation between LRC apoptosis and priming may arise from the coordination between root cap apoptosis and meristem growth dynamics, consistent with a recent study demonstrating this coordination (Shi et al., 2018) and the observed persistence of 
priming in the apoptosis defective smb mutant (Xuan et al., 2016). Finally, findings indicating that mutations in pericycle specific cell cycle regulators affect the frequency of LR formation corroborate the importance of cell growth and division dynamics in determining LR formation (Nieuwland et al., 2009).

Lateral roots are an important determinant of both dicot taproot and monocot fibrous root architectures, Available data indicate a common pericycle origin of these lateral roots, while depending on the species also cortical and endodermal cell layers may contribute to lateral root formation(Xiao et al., 2019) . Intriguingly, depending on the species the auxin maxima preceding lateral root formation may form at either the protoxylem (e.g. Arabidopsis) or protophloem (e.g. maize) vascular pole (Jansen et al., 2012), possibly as a result of differently oriented radial auxin transport. In either case formation of the auxin maxima critically depends on auxin transport, and vascular priming signals are transmitted to the pericycle. Combined with the fact that the priming mechanism uncovered here depends on the basics of stem cell driven root growth, that is shared between all higher, true roots containing plants, this suggests that while radial patterning may be species specific, oscillatory longitudinal auxin patterning is likely largely conserved. Indeed, our model results indicate that changes in root tip size, shape and number or number of cortical layers do not change priming dynamics (Suppl. 3: Fig. 9).

In summary, our work presents evidence that LR priming is driven by a non-canonical mechanism for periodic pattern formation that is distinguishable from other known periodic patterning mechanism such as a clock-and-wavefront model or a Turing pattern.

\section{Limitations of this study}

A major limitation of the current study is that the lateral root priming mechanism we uncovered in silico could not be experimentally validated in a direct manner and instead required a more indirect validation of model predictions. Firstly, direct validation of the priming mechanism through perturbation would require interfering with either the dynamic formation of periodic cell size differences or with the cell size dependence of auxin uptake. It is to our knowledge practically impossible to in planta perturb the relationships between time available for growth and attained cell size, or of cell surface area with passive uptake capacity in a targeted, systematic manner (note that we did perform these experiments in silico, confirming our hypothesis (Supplement 1)). Secondly, an alternative direct validation of the priming mechanism would be to experimentally demonstrate a strong correlation between the periodic formation of large-small cell pairs in the early EZ and auxin oscillations or subsequent lateral root formation. However, this would require the dynamic monitoring of transient cell size differences either simultaneous with auxin dynamics or combined with tracing these to lateral root development. Again, to our knowledge it is currently not feasible to dynamically measure vascular cell sizes due to the narrow shape and internal tissue localization of the vasculature.

\section{Acknowledgments}

We thank Joop Vermeer for financial support of K.Y. Rutger Hermsen for advice on statistical analysis and Jan Kees van Amerongen for management of computational facilities.

\section{Funding}

TvdB and KtT were funded by grant nr 864.14.003 of the Dutch Organization for Scientific Research (Nederlandse Organisatie voor Wetenschappelijk Onderzoek, NWO), H.G and T.B by a grant G022516N of The Research Foundation - Flanders (FWO), J.S.T was funded by grant nr 737.016.012 of NWO, K.Y is funded by VIDI grant nr 864.13.008 of NWO.

\section{Author contributions}

TvdB, KtT, HG, TB, KY, VW and BS wrote the manuscript. KtT and BS conceived the study. TvdB build the model, performed model simulations. TvdB, KY and JST, and analyzed experimental data. KY, VW and HG performed the experiments.

\section{Declaration of Interests}

The authors declare no competing interests. 


\section{Figure legends}

Fig. 1 Auxin oscillations automatically emerge from a growing root model.

A) Overview of root model with tissue types (left) and division, expansion and differentiation zones (right).

B) Model auxin dynamics. Top: Kymograph displaying snapshots of auxin levels in a longitudinal line inside the vasculature at 100s intervals, Bottom: Auxin levels in a high (red line) and a subsequent low auxin (cyan) experiencing cell as a function of time(left) and space (right), cells were followed from entrance in the TZ to exit to DZ.

C) Spatial auxin profile for different auxin production settings for a cell experiencing high (solid line) and low (dotted line) auxin. Auxin production settings were altered to obtain a similar reduction in total auxin for the 3 different cases.

D) Same spatial auxin profile for different auxin production settings as shown in $\mathrm{C}$ but now with auxin levels normalized against total root tip auxin content.

E) Spatial auxin profile for altered reflux loop settings, for a cell experiencing high (solid line) and low auxin (dotted line) with cellular auxin levels normalized for total auxin content. Simulations shown are default simulation (red, as shown in B), simulation with only downward PIN1 in vasculature (yellow), 50\% reduction in PIN2 in LRC, epidermis and cortex without changing the relative strength of basal and lateral transport (purple), absent lateral PIN in LRC, epidermis and cortex from TZ shootward (cyan) and an aux1 mutant with a 90\% reduction in AUX/LAX geneexpression (green).

\section{Fig. 2 Role of root cap and vascular growth dynamics in priming.}

A) Normalized temporal auxin dynamics for a simulated smb mutant and absence of LRC growth. B) Kymograph for a simulation in which only vasculature and pericycle grow. Auxin scale is normalized to maximum observed auxin levels, in Fig. S2B the same scale as in Fig. 1B is used C) Normalized auxin dynamics for reduced width of vasculature cell files. This simulation with decreased vasculature cell width was performed with 1 extra vasculature file to avoid simultaneously altering overall vascular tissue width and hence root architecture..

D) Auxin dynamics for relative to cell height normalized active auxin import (AUX1), active export (PIN) or passive import. Normalization of transport for cell height was applied to vasculature cells in the TZ, EZ and DZ. Cellular auxin levels were normalized for total auxin content to allow comparison between the different simulations.

E) Normalized auxin dynamics for tissue specific TZ onset of the vasculature and pericycle, only cells experiencing high auxin are shown. Arrows indicate the spatial position of the auxin maximum.

F) Root zonation (left) and kymographs of vasculature (upper) and pericycle (lower) for the same simulation with tissue specific TZ onset as shown in E. Cyan arrows indicate priming onset in vasculature and transmission to neighboring pericycle.

\section{Fig. 3 Priming arises in abutting large-small cell pairs.}

A) Kymograph showing auxin (upper) and cell sizes (lower), green dot indicates a large cell followed by a small cell and blue dot a large cell followed by another large cell.

B) Maximum auxin levels as a function of cell height upon TZ entrance for simulation shown in A. Cells entering the EZ between 10-30h of simulation time are shown. Colors indicate the time spent in the EZ until the maximal auxin level is reached. Circle size indicates the ratio between the cell's size and that of the cell below it.

Fig. 4 Priming frequency and amplitude as a function of growth parameters.

A) Simulation data of priming frequency as a function of TA division rate for meristem sizes between $25-30$ or 30-35 cells. Per meristem range and TA division rate, SC division rates were varied between 0.2-0.67 times TA division rate. Data were fitted using linear regression. 
B) Simulation data of priming frequency as a function of meristem size, for cell cycles of $12 \mathrm{~h}$, $9 \mathrm{~h}$ or $8 \mathrm{~h}$ (corresponding to division rates of $0.083,0.11$ and 0.125 , respectively). SC division rates were varied as in A. Data were fitted to a power-law distribution.

C) Simulation data of priming amplitude as a function of elongation time.

D) Simulation data of priming frequency as a function of elongation time for the same simulations as shown in $\mathrm{C}$. See legend next to panel $\mathrm{D}$ for the elongation time corresponding to the different line colors.

\section{Fig. 5 Priming arises from asynchronous clones with final divisions in a limited spatio-} temporal window.

A) Clone number as a function of $\log _{2}(M Z$ size). Data were fitted using linear regression

B) Clone size (blue) and density (red) as a function of MZ size. Data were fitted using linear regression.

C) Zoomed in kymograph showing that cells with a final division in the indicated spatial window arrive large in TZ, and when followed by a small, recently divided cell obtain highest auxin levels. Window is indicated as area between horizontal green lines.

D) Size of window in which final divisions lead to priming for incrementally increasing $M Z$ sizes, from left to right 15, 25 and 35 cells in MZ. Cyan cells indicate cells divided in window followed by a recently divided cell (indicated green).

E) Number of divisions within window that lead to priming for incrementally increasing TA division rates, from left to right $0.08 \mathrm{div} / \mathrm{cell} / \mathrm{h}$ (cell cycle $=12 \mathrm{~h}$ ), $0.11 \mathrm{div} / \mathrm{cell} / \mathrm{h}$ (cell cycle $=9 \mathrm{~h}$ ) and 0.14 $\mathrm{div} / \mathrm{cell} / \mathrm{h}$ (cell cycle $=7 \mathrm{~h}$ ).

Fig. 6 PS spacing and frequency as a function of division rate and MZ size.

A) Simulation data for priming site spacing as a function of $M Z$ size. Data were fitted using linear regression.

B) Simulation data for PS spacing as a function of division frequency. Data were fitted using linear regression was performed.

C) Simulation data for priming frequency as a function of cell production for simulations with varying meristem size and division frequency. Data were fitted using linear.

D) Simulation data for priming site spacing as a function of cell production for simulations with varying meristem size and division frequency. Data were fitted using linear regression.

E) Predicted relationship for priming frequency with cell production for reflux-and-growth, Turing pattern and clock-and-wavefront models.

F) Predicted relationship for PS spacing with cell production for reflux-and-growth, Turing pattern and clock-and-wavefront models.

G) Experimentally measured cell production as a function of meristem size. Data points from both control and 3 different hormone treatments are shown, with color indicating the treatment.

$\mathrm{H}$ ) Experimentally measured priming frequency as a function of priming site spacing, from the same experiments as shown in G. To measure priming dynamics, plants carrying a DR5:LUC construct were used.

Fig. 7 Priming frequency and PS spacing as a function of cell production.

A, B) Experimentally measured priming frequency (A) and PS spacing (B) as a function of cell production. Data points from both the control and the three different hormone treatments are shown, now without using different colors to differentiate treatments. To measure priming dynamics, plants carrying a DR5::LUC construct were used.

$C, D)$ Priming frequency (C) and PS spacing (D) as a function of cell production in simulations incorporating cell type specific zonation, increased vascular division rate and cell height.

E,F) Number of LRs per day (E) and LR spacing (F) as a function of cell production. Data points from both the control and the three different hormone treatments are shown. To measure LR formation dynamics, plants carrying a PLT3::GUS construct were used.

Data in A-F were fitted using linear regression.

Fig. S1 Auxin oscillations automatically emerge in a growing root model. 
A) Model output for default parameter settings, from left to right: snapshots of AUX/LAX and PIN membrane patterns and cellular auxin levels; Color scale indicates relative levels of these properties.

B)Kymographs for pericycle, vasculature, epidermis and endodermis auxin levels for default parameter settings. Color scale indicates cellular auxin levels for all 4 kymographs.

C) Snapshot of auxin pattern at $26 \mathrm{~h}$ simulation time and (vasculature) kymographs for reduced auxin availability.

D) Snapshot of transporters (PIN or AUX/LAX) and auxin patterns at 26h simulation time and kymographs for simulations with altered reflux loop settings. Auxin color bars were scaled per simulation for visualization purposes.

Fig. S2 Role of root cap and vascular growth dynamics in priming.

A) Kymographs of vascular auxin levels for a simulated smb mutant and absence of LRC growth.

B) Kymographs for simulations without growth in epidermis (left), only growth in vasculature and pericycle (middle) and only growth in epidermis, cortex and endodermis (right). For comparison purposes, the auxin scale bar is kept constant relative to the simulation in which all tissues grow (Fig 1B).

C) Simulation data for a simplified 1D model. From left to right: schematic depiction of the developmental zonation, PIN1 pattern and lateral auxin influx mimicking a reflux loop that generates an auxin loading zone at the MZ/EZ boundary (tissue width is amplified for ease of visualization), kymograph for 1D model without lateral auxin influx and kymograph for 1D model incorporating lateral auxin influx.

\section{Fig. S4 PS spacing and frequency as a function of division rate and MZ size.}

$A, B)$ Simulations performed with varying division rate $(A)$ or varying $M Z$ size $(B)$ while keeping stem cell division constant.

Fig. S6 Distribution of measurements for growth and priming parameters

A-D) Boxplot of experimental measurements for $M Z$ size $(A)$, cell production (B), priming frequency $(C)$, and priming site spacing (D) and cell division rate (G). Color indicates data set, all data combined (brown, $n=104$ ), control/MS (blue, $n=30$ ), 10 $\mu$ M GA (yellow, $n=26$ ), $1 \mathrm{nM}$ BR (red, $n=31$ ) and $100 \mathrm{nM}$ BR (green, $n=17$ ). Boxes cover lower to upper quartile values, black line indicates median and white square mean, whiskers indicate the total range of the data and black dots indicates outliers.

Fig. S7 Priming frequency and spacing as a function of cell production.

$A, B)$ Experimentally measured priming frequency (A) and PS spacing (B) as a function of cell production. Data points are colored to indicate the different treatments.

C) Experimentally measured priming frequency as a function of division rate. To avoid confounding effects, subsets of data containing only plants with a similar MZ size were used. Data points from both the control and the three different hormone treatments are used.

D) Experimentally measured priming frequency as a function of meristem size. To avoid confounding effects, subsets of data containing only plants with a similar division rate were used.

Data points from both the control and the three different hormone treatments are used.

E) Priming site spacing as a function of MZ size for experiments. Data points are colored to indicate the different treatments.

F) PS spacing as a function of division frequency for experiments. Data points are colored to indicate the different treatments.

$\mathrm{G}, \mathrm{H})$ Model priming frequency $(\mathrm{G})$ and PS spacing $(\mathrm{H})$ as a function of cell production. Vasculature specific details were incorporated into the model alone or in combination, and results were compared to those for default model settings (green line).

In $A, B, E, F$ the combined data of different treatments were fitted to a single linear regression line. In $\mathrm{C}, \mathrm{D}, \mathrm{G}, \mathrm{H}$, data was fitted using linear regression per color-indicated subset.

Video S1: Auxin oscillations automatically emerge in a growing root model.

Growing root model with cellular auxin levels (left), 1 pixel wide snapshots of the root's vasculature are displayed in the kymograph (right) over the time course of the simulation. 
Video S4a: Priming frequency increases with increasing division rate.

Root simulations with varying cell cycle duration (inverse of division rate) while keeping meristem size constant. From left to right: cell cycle $6 \mathrm{~h}$ (division rate $=0.1667 \mathrm{~h}^{-1}$ ), cell cycle $9 \mathrm{~h}$ (division rate $\left.=0.11 \mathrm{~h}^{-1}\right)$ and cell cycle $12 \mathrm{~h}\left(\right.$ division rate $\left.=0.183 \mathrm{~h}^{-1}\right)$.

Video S4b: Priming frequency increases with increasing meristem size.

Root simulations with varying meristem sizes while keeping division rate constant. From left to right: 12 meristem cells, 25 meristem cells and 40 meristem cells. Note that meristem cell counts only represent actively dividing cells, TZ cells were not counted as part of the meristem. Also note that for increasing MZ sizes in our model oscillation amplitude decreases. This is due to the reduced time for elongation in the loading zone resulting from the higher cumulative displacement caused by larger cell numbers. In planta, elongation rates appear to be compensated for higher division rates or meristem sizes, reducing this effect.

Video S4c: Priming amplitude but not frequency is influenced by elongation rate.

Root simulations with varying elongation durations (inverse of elongation rate) while keeping cell cycle time and meristem size constant. From left to right: elongation time $5.5 \mathrm{~h}$ (elongation rate = $0.18 \mathrm{~h}^{-1}$ ), elongation time $7.5 \mathrm{~h}$ (elongation rate $=0.13 \mathrm{~h}^{-1}$ ) and elongation time $9.5 \mathrm{~h}$ (elongation rate $\left.=0.105 \mathrm{~h}^{-1}\right)$. 


\title{
STAR METHODS
}

\author{
RESOURCE AVAILABILITY
}

\section{Lead Contact}

Further information and requests for resources and reagents should be directed to and will be fulfilled by the Lead Contact, Kirsten ten Tusscher (k.h.w.j.tentusscher@uu.nl).

\section{Materials Availability}

This study did not generate new unique reagents or materials

\section{Data and Code Availability}

The code and datasets generated during this study are available at

[http://bioinformatics.bio.uu.nl/khwjtuss/PrimingRoot].

\section{EXPERIMENTAL MODEL AND SUBJECT DETAILS}

Plant material

Arabidopsis thaliana accession Col-0 was used in this work. The transgenic Arabidopsis thaliana lines used were described previously: pPLT3::GUS (Du and Scheres, 2017) and DR5::LUC (Moreno-Risueno et al., 2010; Xuan et al., 2016). Seeds were surface sterilized with gaseous chlorine produced in a sealed container with $80 \mathrm{ml}$ bleach supplemented with $3 \mathrm{ml}$ of $37 \%$ hydrochloric acid for 2 hours. Seeds were sown on growth medium consisting of half-strength Murashige Skoog salts (1/2 MS) with $1-1.5 \%$ agar for lateral root number assay and luciferase assay respectively. Seeds were then stratified at $4^{\circ} \mathrm{C}$ for 2 days in the dark and transferred to growth chamber at $22^{\circ} \mathrm{C}$ for germination under long day conditions ( $16 \mathrm{~h}$ light/ $8 \mathrm{~h}$ dark photoperiod) in oriented Petri dishes (15 degree to vertical plane).

\section{METHOD DETAILS \\ Experimental methods}

\section{Hormone Treatments}

Seeds were sterilized, plated and seedlings were grown as described in the plant material subsection. The seedlings were transferred with tweezers onto solid $1 / 2$ MS medium plates containing Gibberellic acid (GA) at a final concentration of $10 \mu \mathrm{M}$ or Brassinosteroid (24Epibrassinolide) (BR) at a final concentration of $1 \mathrm{nM}$ or $100 \mathrm{nM}$. As control the seedlings were transferred to $1 / 2$ MS medium without any supplements. For the lateral root number assay the total duration of the hormonal treatment was 6 days. Quantification of lateral roots was performed over a 4 day period, starting 2 days after transfer to the treatment plates to avoid measuring initial, transfer induced stress response rather than hormonal responses. For the luciferase assay the hormonal treatment period was 66 hours, Luciferase imaging of the root began 48 hours after transfer to the treatment plates (more details can be found in the respective sections below).

\section{Histology and Microscopy}

Histochemical staining of promoter-driven $\beta$-glucuronidase (GUS) activity was performed as described previously (Willemsen et al., 1998) with modifications. In brief, a strip of filter paper soaked in GUS staining solution $(0.5 \mathrm{mg} / \mathrm{ml}$ X-gluc dissolved in $\mathrm{n}$-dimethyl-formamide, $0.1 \%$ Triton $\mathrm{X}-100,0.5 \mathrm{mM} \mathrm{K} 4 \mathrm{Fe}(\mathrm{CN}) 6 . \mathrm{H} 2 \mathrm{O}, 0.5 \mathrm{mM} \mathrm{K3Fe}(\mathrm{CN}) 6$, and $50 \mathrm{mM}$ sodium phosphate buffer, $\mathrm{pH}$ 7.2) was placed on the root section of interest and incubated at $37^{\circ} \mathrm{C}$ in the dark for 2 hours. Stained primordia were counted under a Carl Zeiss Stemi SV6 Stereomicroscope. For meristem and cortical cell size analysis, roots were stained and fixed in SCRI Renaissance 2200 Staining Solution $(0.1 \%(\mathrm{v} / \mathrm{v})$ SR2200, 1\% (v/v) DMSO, 0.05\% (w/v) Triton X-100, 5\% (w/v) glycerol, 4\% $(\mathrm{w} / \mathrm{v})$ para-formaldehyde in PBS buffer $(\mathrm{pH} 8.0)$ and stored at $4^{\circ} \mathrm{C}$ before imaging as described previously (Kerstens et al., 2020). Images were acquired using a Zeiss LSM 710 confocal laser 
scanning microscope with 40X objective with laser lines and setting as described previously (Kerstens et al., 2020). Images were analyzed with Fiji (Schindelin et al., 2012).

\section{Lateral root number assay}

PLT3::GUS seedlings grown on $1 / 2$ MS plates for 5 days were transferred onto respective treatment/control plates. 2 days after transfer, the root tips of the seedlings were labelled and the plates were scanned using Epson Expression 11000XL every 24hrs for 4 consecutive days. Displacement of root tips indicated by consecutive marks was used to determine root growth per day. At the end of the treatment period, the number of lateral root primordia/ emerged lateral roots formed per day were quantified by counting the GUS stained primordia in each labelled section.

Luciferase assay

DR5::LUC seeds were plated on $1 / 2 \mathrm{MS}$ medium and grown for 5 days before transferring onto compartmented plates (Nunc ${ }^{\mathrm{TM}}$ Thermo Scientific ${ }^{\mathrm{TM}}$ ) containing two hormonal treatments randomly selected from a paired design. The next day, we sprayed the seedlings with a $1 \mathrm{mM} \mathrm{D}$-Luciferin solution (dissolved in $0.01 \%$ Tween $80,0.1 \%$ DMSO) in order to reveal their DR5::LUC expression. 24 hours after spraying in vivo luminescence signal was monitored for 18 hours using the Nightshade LB 985 (Berthold) system adapted for live-imaging of vertically growing Arabidopsis seedlings by (Xuan et al., 2018).

Similar to an approach previously followed by Kircher and Schopfer, we measured in vivo DR5:LUC luminescence in plants exposed to intermittent periods of darkness (allowing luminescence recording) and light (simulating normal growth chamber conditions)(Kircher and Schopfer, 2018). Specifically, for a period of $18 \mathrm{~h}$ we applied 22 min cycles in which plants were exposed to $14 \mathrm{~min}$ light, and to 8 min of darkness, recording DR5::LUC expression for the final 3 min of darkness, DR5::LUC expression was recorded using an integrated charge-coupled device (CCD) camera, acquired the emitted signal with a 5 minutes exposure time and a $2 \times 2$ binning resolution. During the 14 minutes of light exposure, we simulated the growth chamber light intensity using the Nightshade LED panels. After imaging roots were stained and fixed in SR2200 Staining Solution to perform analysis on primary root meristem.

\section{Kymograph analysis}

Stack images from microscopy analysis were analyzed by making a kymograph using the KymoResliceWide plugin for FiJi (https://github.com/ekatrukha/KymoResliceWide). A line matching the width of an individual root was drawn on the root in the last time frame of the movie, the plugin was used with 'average' settings to avoid temporal differences in the stacks to influence the outcome of the analysis. A space scale was added while processing the kymograph (Methods Fig. 1A).

Image J/Fiji cannot handle different scales in $\mathrm{x}$ and $\mathrm{y}$ direction therefor only a space scale was added, while a time scale was manually added based on the duration of the experimental recording. Brightness and contrast was adjusted per individual root to allow visual identification of priming events. Since no conclusions are drawn from or analysis is based on priming amplitudes, adjusting settings per individual root did not affect our analysis of priming frequency and spacing.

\section{Kymograph analysis, root growth}

To measure root growth rate over the $18 \mathrm{~h}$ period of the recording a line was drawn perpendicular to the position of the root tip at the end of recording (Methods Fig. 1B, red line). Subsequently, a straight line was drawn from this first line to the position the root tip occupied at the start of the recording (Methods Fig. 1B, cyan line), with the total size of this line representing overall root tip displacement and hence overall root growth. Dividing this root growth over the time of the recording results in a root growth rate. Root growth rate measurements were afterwards divided over the average adult (expanded) cell size of the respective root to obtain root growth rate expressed in cell numbers/h.

\section{Kymograph analysis, priming site spacing}

To determine priming site spacing a line was drawn corresponding to the position of the $t=18 \mathrm{~h}$ time frame (Methods Fig. 1C, yellow dotted line), using a line width of 3 pixels to enhance resolution. 
Start and end position of this line are determined by the position of the TZ/EZ border (location where priming occurs, recognizable as the second diagonal line from the left in the kymograph) at the start (Methods Fig. 1C, red line) and end of the recording period. We have thus defined, for a particular time point, a spatial domain in which we can determine the spacing of priming events.

To determine spacing, we plot DR5:LUC intensity along the defined spatial domain (Methods Fig. 1D). To measure actual priming site spacing rather than pre-branch site spacing priming events that do not result in maintained high pixel intensity but instead fade out were artificially enhanced with a white line to allow measurement at the $t=18 \mathrm{~h}$ position in the kymograph (Methods Fig. 1D).

A intensity plot over space was obtained and was used to determine the priming site spacing (Methods Fig. 1E). Priming site spacing (PSS) per root was calculated in the following way:

PSS $(\mathrm{mm})=\left(\right.$ peak $_{\text {last }(\mathrm{mm})}-$ peak $_{\text {first(mm) }}-$ peaks $_{\text {num }}{ }^{*}$ size $\left._{\text {cell }}\right) /\left(\right.$ peaks $\left._{\text {num }}-1\right)$

Where peak $\mathrm{k}_{\text {last(h) }}$ and peak $\mathrm{k}_{\mathrm{first}(\mathrm{h})}$ are the positions in space of the last and first DR5::LUC intensity peak respectively, peaks num are the number of peaks counted and size cell refers to the average adult cell size of the respective root. The - peaks ${ }_{\text {num }}{ }^{*}{ }$ size $_{\text {cell }}$ serves to substract from the total distance between first and last priming event the space occupied by priming events themselves, thus restricting inter priming distance calculation to in between non-primed cells. The above formula calculates the PSS in $\mathrm{mm}$, by dividing this distance afterwards over adult cell size of the corresponding root we compute PSS in cell numbers.

\section{Kymograph analysis, priming frequency}

To independently determine priming frequency we need to determine the number of priming events occurring along the time axis. However, since the resolution of the time axis is considerably lower than that of the space axis, projecting priming events on the time-axis will likely results in highly noisy priming frequency data. As an alternative approach, to make use of the higher resolution in the spatial dimension, we first draw a diagonal line following the position of the root tip over time (Methods Fig. 1G, yellow line), We set the scale of this spatio-temporal root tip trajectory line to the $18 \mathrm{~h}$ of the recording period. Next, we draw a line parallel to this line, corresponding to the displacement over time of the end EZ/start DZ where priming events are most clearly visible (Methods Fig 1G, yellow dashed line). The time scale of this line ( $\mathrm{mm}$ to $\mathrm{h}$ conversion) is obtained from the root tip line. We have thus defined, for a particular spatial trajectory, a temporal domain in which we can determine priming frequency.

To determine frequency DR5:LUC intensity was plotted along the defined temporal domain, again fading out priming events where artificially enhanced to determine frequency of priming rather than pre-branch site formation (Methods Fig. 1F). 

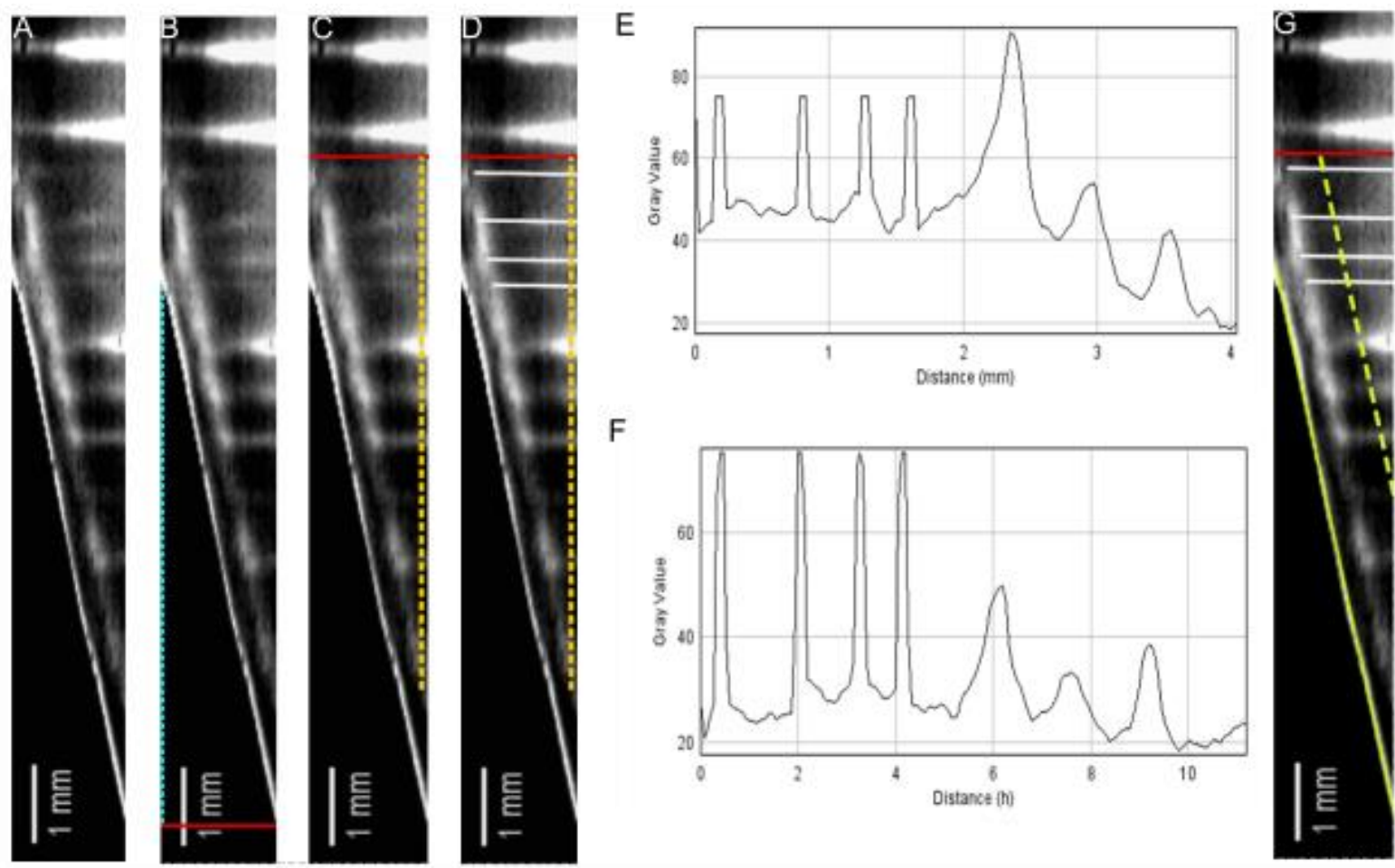

Methods Figure 1. Kymograph analysis experimental results. A) Example kymograph B) Growth was measured through a line (cyan) running from the location of the root tip at the start to the end of the imaging period (red line indicates root tip location at the end of imaging). C) PSS was determined by measuring intensity on a line matching the last time frame (18h) of imaging (dotted yellow line), a line perpendicular to the location of the TZ at the start of imaging was taking as the start of the measurements. D) To account for all priming events, fading priming events where enhanced with a white line. E) Intensity values along the yellow line shown in $D$ for analysis of the spacing of the primed sites. F) Intensity values along the yellow line in $G$ for analysis of the timing of priming events. G) Priming frequency was measured by obtaining a spacetime scale from the root tip growth (solid yellow line) and measuring intensity from a line in the EZ exactly parallel to the root tip. TZ location at the start of imaging (red line) was taken as the start of measurements.

The priming period (inverse of priming frequency) per root was calculated as follows:

Priming period $(\mathrm{h})=\left(\right.$ peak $_{\text {last( }(\mathrm{h})}-$ peak $\left._{\text {first( }(\mathrm{h})}\right) /\left(\right.$ peaks $\left._{\text {num }}-1\right)$

Where peak last(h) and $_{\text {peak }}$ first (h) $_{\text {are }}$ the occurrence in time of the last and first DR5:LUC intensity peak respectively, peaksnum are the number of peaks counted.

In total $n=132$ roots were grown and imaged (MS, $n=33 ; 10 \mu m$ GA, $n=31 ; 1 \mathrm{~nm} B R \mathrm{n}=32$ and 100nm BR $n=36$ ), $n=104$ were analysed. Data from 28 roots was excluded from analysis due to various reasons: growing out of the imaging plane $(n=5)$, heavy curling $(n=3)$, curling and touching of neighboring root $(n=14)$, lack of growth $(n=2)$, lack of resolution $(n=4)$. Roots treated with $100 \mathrm{~nm}$ $B R$ were more prone to curling and hence touching of neighboring roots. Of the total of $n=28$ excluded roots, $n=19$ were treated with $100 \mathrm{~nm} B R, n=5$ with $10 \mu \mathrm{m} \mathrm{GA}, \mathrm{n}=1$ with $1 \mathrm{~nm} B R$ and $n=3$ were on control medium.

\section{Measuring active meristem size}

Meristem imaging was done as mentioned in the histology and microscopy section. To measure meristem size and mature cortical cell sizes, Fiji/lmage $\mathrm{J}$ was used (Schindelin et al., 2012), applying the Cell-0-tape macro for cell size measurements (French et al., 2012). Previous research has demonstrated that due to the stopping of cell division and onset of cell elongation, the boundary of the actively dividing meristem corresponds to the position in graph plotting cell size as a function of distance from the QC where cell size increase starts to accelerate (Hayashi et al., 2013; Ivanov and Dubrovsky, 2013). Therefore, to determine active meristem size, we plot cortical cell size as a function of position, doing this for both cortical cell files. We defined the meristem boundary as the position where the slope of cell size changes from neutral to positive (Methods 
Fig.2 green line). If we obtain different meristem boundary positions from the two cortical cell files, an average meristem boundary position was computed.

All $n=104$ roots that were used for kymograph analysis were also used for active meristem size measurements.

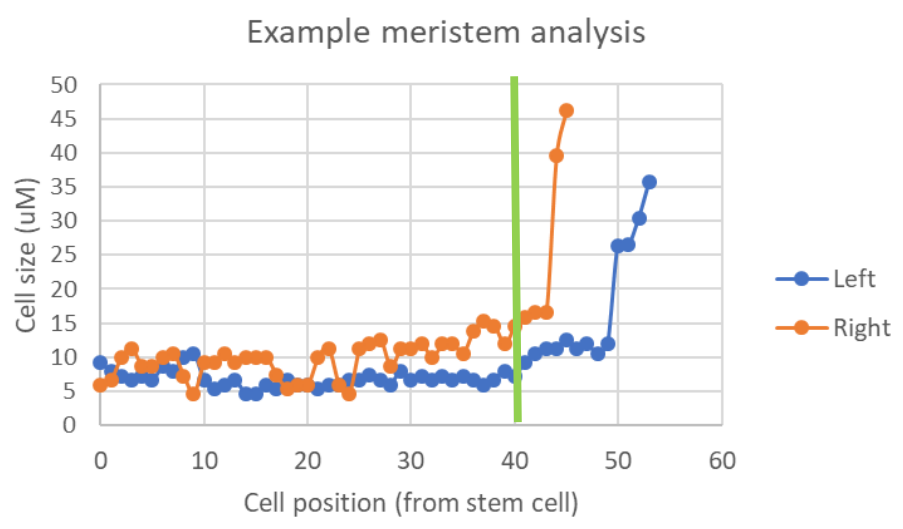

Methods Figure 2. Example meristem analysis, cell size from two cortical files on either side of the root were measured and plotted against their position from the stem cell. The point where in both cortical cell files cell size start to increase was taken as a measure of the end of the active meristem.

\section{Determining cell production and division frequency}

To determine (cortical) cell production rate, the number of (cortical) cells produced by the meristem per hour, we divide the measured root growth rate (in $\mu \mathrm{m} / \mathrm{h}$ ) (see section Kymograph analysis, root growth) over the mature cortical cell size (in $\mu \mathrm{m}$ ).

Next, to determine (cortical) cell division frequency, the number of cell divisions per hour, we divide the obtained cell production rate (in cells/h) over the number of meristematic cortical cells (see section Measuring active meristem size).

\section{Computational methods}

\section{General model description}

We developed a novel multi-scale model for root growth and development, using as a basis a combination of our earlier root models that either incorporated a realistic root tip architecture (van den Berg et al., 2016) or root growth dynamics (Mahonen et al., 2014), similar to what we recently published (Salvi et al., 2020). As a critical extension thereof, we incorporated in more detail root meristem activity, including realistic cell division patterns with slow stem cell (SC) divisions near the quiescent center (QC) and subsequent clonal expansion of more rapidly dividing transit amplifying (TA) cells (Fig. 1A) (Bizet et al., 2015; Rahni and Birnbaum, 2019). Like our earlier models, the model incorporates cell type specific and zonation dependent gene expression and polarity patterns of AUX/LAX auxin importers and PIN exporters (Fig S1A), developmental zone specific cellular growth, division, expansion and differentiation dynamics (Fig. 1A), cell level control of gene expression, and sub cellular, grid level, simulation of auxin dynamics. With respect to gene expression, the model only incorporates the auxin-dependent gene expression of AUX/LAX.

\section{Tissue lay-out}

In the current study we aimed to investigate the interplay between auxin transport and root growth dynamics. Work by us and others has demonstrated the importance of a realistic root tip layout, as compared to a simplified rectangular root topology, for root tip auxin patterning (Cruz-Ramirez et al., 2012; van den Berg et al., 2016). Thus we need to incorporate in our model an anatomically realistic, wedge-shaped root tip layout encased in a lateral root cap (LRC). At the same time, our research goal requires the incorporation of root growth dynamics. However, since the development of a full mechanical model of root growth dynamics is outside the scope of the present paper, the aim was to use the previously applied simplistic method of simulating root growth dynamics in 
which cells grow by adding a row of grid points and shifting upward all more shootward cells (Mahonen et al., 2014). While this root growth algorithm can be easily applied in a square root topology in which all cells are stacked in straight columns, this approach is less easily extended to the curved regions of the root tip. Therefore, as a compromise, we limited the size of the curved part of our root topology and ignored cell growth and divisions there, simulating growth dynamics only in the straight part of the root architecture. We reasoned that this is a justified approximation since it only ignores growth dynamics of the columella and lowermost parts of the RC, which do not contribute to root tip meristem growth dynamics. The root layout was simulated on a grid of $224 \times 2255 \mu \mathrm{m}^{2}$ with a spatial resolution of $2 \mu \mathrm{m}$. A total of 8 different cell types were incorporated in the model, with cell type specific widths incorporated based on experimental data and earlier modelling studies (Laskowski et al., 2008; van den Berg et al., 2016). In figure 1A, left side of root, moving from outermost to innermost these are: RC and LRC (maroon, $8 \mu \mathrm{m}$ in straight part root), epidermal (blue, $18 \mu \mathrm{m}$ ), cortical (light green, $20 \mu \mathrm{m}$ ), endodermal (orange, $12 \mu \mathrm{m}$ ), pericycle (yellow, $8 \mu \mathrm{m}$ ) and 3 vasculature files (dark green, $6 \mu \mathrm{m}$ ). Finally, the vasculature converges on the QC (pink) and below the QC are the columella cells (grey) (Fig. 1A).

To simulate developmental zonation dynamics, our model root is subdivided into 4 distinct developmental zones, moving from the root tip shootward these are: meristematic zone (MZ), with cytoplasmic growth and cell division; transition zone (TZ), with cytoplasmic growth but without further cell division; elongation zone (EZ), with vacuolar expansion; and differentiation zone (DZ), in which cells undergo terminal differentiation without growing further (Fig. 1A, right part of root). In the model used in this study, to simplify matters, the position of zonation boundaries were defined in terms of distance from the QC rather than made dependent on auxin (Grieneisen et al., 2007), PLT (Mahonen et al., 2014) or combined PLT and cytokinin signaling (Salvi et al., 2020) gradients. Boundary positions were set such that the combined meristem and transition zone contains an average number of 40 cells for default simulations (average size 1.5 times the $8 \mu \mathrm{m}$ they have just after division). The rootward $75 \%$ of these cells belong to $M Z$ and will proliferate while the shootward $25 \%$ belong to the TZ, exhibiting cytoplasmatic growth but no longer dividing. The EZ contains between 7-10 cells with a height between 20 and $174 \mu \mathrm{m}$ and the DZ contains 8-12 cell with an size of $175 \mu \mathrm{m}$. PIN expression and polarity patterns as well as AUX1/LAX patterns where incorporated based on tissue type and developmental zone, in agreement with experimental data (Bennett et al., 1996; Péret et al., 2012; Swarup et al., 2001; Swarup et al., 2005) and similar to earlier modeling studies (Grieneisen et al., 2007; Laskowski et al., 2008; Mahonen et al., 2014; Salvi et al., 2020) (Fig. S1A). This pattern of auxin transporters results in reverse fountain auxin reflux pattern with maximum levels in the QC (Grieneisen et al., 2007) (Fig. S1A).

\section{Auxin dynamics}

Auxin metabolism, passive and active transport across the membrane, and intracellular and intraapoplast diffusion were implemented on a subcellular, grid point level in a similar manner as in earlier studies(Grieneisen et al., 2007; Mahonen et al., 2014; van den Berg et al., 2016).

For a cytoplasmic grid point $\mathrm{i}, \mathrm{j}\left(\mathrm{A}_{\mathrm{i}, \mathrm{j}}\right)$ surrounded by $n$ wall $\left(\mathrm{A}_{\text {wall }}\right)$ and $m$ cytoplasmic $\left(\mathrm{A}_{\text {cell }}\right)$ grid points the equation is as follows:

$\frac{\partial A_{i, j}}{\partial t}=p_{A}+D_{\text {cell }} \frac{\partial^{2}}{\partial x^{2}}\left(\sum_{l=0}^{m} A_{c e l l}-A_{i, j}\right)+\sum_{k=0}^{n}\left(\left(i_{\text {pas }}+\frac{i_{A U X}}{L A X}\right) A_{\text {wall }, k}\right)-\sum\left(\left(e_{P I N}+e_{b}\right) A_{\text {wall }}\right)-$ $d_{A} A_{i, j}(1)$

Here, $p_{A}$ is the auxin production rate, $d_{A}$ is the auxin degradation rate, and $D_{c e l l}$ is the diffusion rate of auxin inside a cell. $i_{\text {pas }+ \text { act }}$ is the combined passive, diffusional and active, AUX/LAX mediated influx of auxin from walls to cytoplasm, $e_{P I N}$ represents active, PIN mediated export of auxin from cytoplasm to walls, and active transport by other not explicitly modeled exporters such as $\mathrm{ABCBs}$ is captured in $e_{b}$. For an apoplastic grid point $\mathrm{i}, \mathrm{j}\left(A_{i, j}\right)$ surrounded by $\mathrm{n}$ wall $\left(A_{\text {wall }}\right)$ and $m$ cytoplasmic $\left(A_{\text {cell }}\right)$ grid points the equation is as follows: 
$\frac{\partial A_{i, j}}{\partial t}=D_{\text {wall }} \frac{\partial^{2}}{\partial x^{2}}\left(\sum_{k=0}^{n} A_{w a l l, k}-A_{i, j}\right)-\sum_{l=0}^{m}\left(\left(i_{\text {pas }+a c t}\right) A_{c e l l, l}\right)+\sum\left(\left(e_{P I N}+e_{b}\right) A_{c e l l}\right)$

With $D_{\text {wall }}$ representing the auxin diffusion rate in the apoplast.

\section{Auxin production}

While historically, root auxin levels were assumed to almost solely depend on shoot delivered auxin, more recent data show the importance of root localized regions of high auxin production, particularly once roots have passed a particular developmental age (Bhalerao et al., 2002). We incorporated elevated auxin production occurring in cells surrounding the QC as well as in the columella and LRC cells (Fig. 1E), assigning these cells with higher values of $p_{A}$ (Method Table 1). Finally, to ensure that despite grid based modeling of auxin dynamics, the overall auxin production of an individual cell is independent of cell size we normalized $p_{A}$ as $p_{A}=\mathrm{p}^{*} \frac{\text { height }_{\text {cell }}}{\text { height }_{M Z \text { cell }}}$.

Where height $t_{c e l l}$ is the actual height of the cell and height ${ }_{M Z c e l l}$ is the initial height of a meristematic cell.

\section{Boundary conditions}

To simulate auxin exchange with the not explicitly modeled shoot, we incorporate an auxin influx (inf) into the top wall of the topmost endodermal and stele cells, while including an auxin efflux (eff) from the top walls of all other cell files with the strength of eff determined by the amount of influx and AUX/LAX on the lower membrane of the top cells. This approach is similar to that used in previous root tip models (Di Mambro et al., 2017; Grieneisen et al., 2007; Mahonen et al., 2014; Salvi et al., 2020; van den Berg et al., 2016)

\section{AUX/LAX pattern}

For simplicity active auxin import was described using a single lumped AUX/LAX import term. To define the lumped expression domain we defined an AUX/LAX prepattern that represents the sum of experimentally reported expression domains of AUX/LAX genes (Bennett et al., 1996; Péret et al., 2012; Swarup et al., 2001; Swarup et al., 2005) (Fig. S1A). Active AUX/LAX mediated influx is described as: $\mathrm{i}_{\mathrm{AUX} / \mathrm{LAX}}=v_{\text {up }}{ }^{*} \mathrm{AUX} / \mathrm{LAX}$ pat ${ }^{*} A U X / L A X_{\text {gen }}$ where $v_{\text {up }}$ is the auxin uptake rate of AUX/LAX, AUX/LAX $X_{\text {pat }}$ is the pre-pattern describing the maximum membrane level of the auxin importers as a function of zone, cell type and membrane face and AUX/LAX $\mathrm{pat}_{\text {it }}$ is the cell level gene expression of AUX/LAX. AUX/LAX expression is auxin dependent (Laskowski et al., 2006; Laskowski et al., 2008), and we recently showed that this auxin dependence plays an important role in root tropisms (van den Berg et al., 2016). Assuming a saturating dependence of AUX/LAX expression on auxin levels we write:

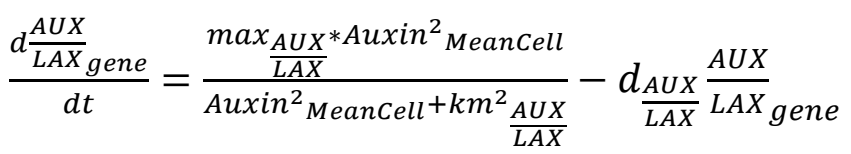

Here, $\max _{\frac{A U X}{L A X}}$ is the maximal gene expression rate of $A U X / L A X, k m_{\frac{A U X}{L A X}}$ is the auxin level at which the rate of AUX/LAX expression is half maximal, AUX/LAX proteins are degraded with rate $\frac{d_{\frac{A U X}{L A X}} \text {, and } A \text { uxin }}{\text { Meancell }}$ is the average cellular auxin level.

\section{PIN expression and localization}

Similar to our earlier studies, we model active auxin export from cells as consisting of a major PIN protein mediated component $\left(e_{\text {pin }}\right)$ and a minor additional component $\left(e_{b}\right)$ that can be thought of as ABCB/PGP mediated auxin export. For simplicity $\left(e_{b}\right)$ is assumed to be equal for all cells and to have an apolar membrane pattern. Similar to $i_{A U X / L A X}$, $e_{P I N}$ is implemented to depend on uptake rate, polarity pattern and gene expression levels in the following way: $e_{\text {PIN }}=v_{\text {out }}$ * PIN $_{\text {pat }}{ }^{*}$ PIN gen where $V_{\text {out }}$ is the rate of PIN mediated auxin transport, PIN pat $_{\text {pat }}$ the PIN pre-patterning describing the maximum membrane level of the auxin importers as a function of zone, cell type and 
membrane face and PIN $\mathrm{Nen}_{\text {gen }}$ the gene expression level of PIN. With regards to PIN mediated transport, tissue type and zonation dependent PIN pre-patterns are incorporated based on experimental data and similar to those used in earlier models (Fig. S1A) (Grieneisen et al., 2007; Laskowski et al., 2008; Mahonen et al., 2014; van den Berg et al., 2016). Previous research has shown the critical importance of protoxylem and xylem pole pericycle in LR priming. The initial priming signal was shown to only occur at the two protoxylem poles, not in other vascular files, and to be transmitted specifically to overlaying pericycle cells in which subsequent LR development occurs (De Smet et al., 2007). Since we aim to model LR priming, we choose for our 2D model to represent a longitudinal cross-section through the protoxylem poles. To achieve this we implemented a vascular PIN pattern emulating critical aspects of the in planta present three dimensional auxin fluxes that result in the directing of auxin towards the protoxylem poles, ensuring protoxylem pole priming only (el-Showk et al., 2015). Specifically, we included in addition to the predominant basally oriented active auxin transport, outward oriented, protoxylem directed PIN transport (Fig. S1A).

Relative to earlier models changes were made in the PIN1 polarity pattern in the MZ, based on recent experimental data demonstrating a relatively apolar distribution of PIN1 in the lowermost regions of the root (Omelyanchuk et al., 2016) (Fig. S1A). This change resulted in a broader, more robust auxin maximum, more consistent with experimentally observed auxin patterns. For simplicity, regulation of and resulting changes in PIN gene expression levels were ignored.

Method Table 1. Auxin parameters

\begin{tabular}{|c|c|c|c|}
\hline Parameter & Description & Value & Dimension \\
\hline$p_{A}$ & Basal auxin production rate & 0.0015 & $\mathrm{~s}^{-1}$ \\
\hline$Q C_{p_{A}}$ & QC auxin production rate & $50^{*} p_{A}$ & $\mathrm{~s}^{-1}$ \\
\hline $\operatorname{Col}_{p_{A}}$ & Columella auxin production rate & $15^{\star} p_{A}$ & $\mathrm{~s}^{-1}$ \\
\hline$L R C_{p_{A}}$ & LRC auxin production rate & $10^{*} p_{A}$ & $\mathrm{~s}^{-1}$ \\
\hline $\inf$ & Shoot influx & 0.96 & $\mu \mathrm{m} \mathrm{s}^{-1}$ \\
\hline$d_{A}$ & Auxin degradation rate & 0.000725 & [auxin] $\mathrm{s}^{-1}$ \\
\hline$D_{\text {cell }}$ & Cytoplasmic diffusion rate & 600 & $\mu \mathrm{m}^{2} \mathrm{~s}^{-1}$ \\
\hline$D_{\text {wall }}$ & Apoplastic diffusion rate & 40 & $\mu \mathrm{m}^{2} \mathrm{~s}^{-1}$ \\
\hline$i_{\text {pas }}$ & Passive influx & 2.5 & $\mu \mathrm{m} \mathrm{s}^{-1}$ \\
\hline Vup & Active influx rate & 0.07 & $\mu \mathrm{m} \mathrm{s}^{-1}[\mathrm{AUX} / \mathrm{LAX}]^{-1}$ \\
\hline $\max _{\overline{A U X}}$ & $\begin{array}{l}\text { Maximal AUX/LAX expression } \\
\text { rate }\end{array}$ & 0.01 & [auxin] $\mathrm{s}^{-1}$ \\
\hline$k m_{\frac{A U X}{L A X}}$ & $\begin{array}{c}\text { Saturation constant AUX/LAX } \\
\text { expression }\end{array}$ & 50 & [auxin] \\
\hline
\end{tabular}




\begin{tabular}{|c|c|c|c|}
\hline$d_{A U X / L A X}$ & AUX/LAX degradation rate & 0.0001 & $\mathrm{~s}^{-1}$ \\
\hline $\mathrm{e}_{\mathrm{b}}$ & Non-PIN mediated export rate & 1 & $\mu \mathrm{m} \mathrm{s}^{-1}$ \\
\hline $\mathrm{V}_{\text {out }}$ & Active efflux rate & 0.2 & $\mu \mathrm{m} \mathrm{s}^{-1}[\mathrm{PIN}]^{-1}$ \\
\hline PIN $_{\text {gen }}$ & Cellular PIN expression levels & 100 & {[]} \\
\hline
\end{tabular}

\section{Growth dynamics}

Earlier data on Arabidopsis root growth dynamics (Beemster and Baskin, 1998) suggested that cell cycle durations in the root meristem (RAM) are in the order of 20 hours. These cell cycle durations were based on measured cumulative cell flux dynamics at the end of the meristem with the assumption that all, approximately 30-35, rows of cells within the meristem divide at a similar rate. In our earlier model, cellular growth dynamics were based on these estimated rates (Mahonen et al., 2014). However, more recent data suggest that cell divisions occur in only a limited, rootward region of the meristem containing 15-20 cell rows (Rahni and Birnbaum, 2019; Wendrich et al., 2017). Cells in the remaining more shootward part of the meristem grow slowly, while not or hardly dividing, until switching to rapid vacuolar expansion driven growth in the elongation zone (Dello loio et al., 2008; Novak et al., 2016). Division rates measured within the lowermost, actively dividing part of the meristem were found up to 3 hours per cell cycle (Campilho et al., 2006; Rahni and Birnbaum, 2019; von Wangenheim et al., 2017). To account for these recent insights, we incorporated in the current model transit amplifying division rates in the range between 8 and 20h. In addition, we also explicitly incorporated a proper meristem zone (MZ) in which cells actively divide and a shootward MZ part, which we will refer to as a transition zone (TZ) in which we ignore rare cell divisions and only simulate slow cytoplasmic cell growth (Fig. 1A).

Individual cells start in the $M Z$ where they grow with rate $r_{\text {growthMz }} / \mu \mathrm{m}$ and divide when they have doubled their size. When leaving the MZ, cells enter the TZ where they still grow with $r_{\text {growthmz }} / \mu \mathrm{m}$ but no longer divide. Upon entering the $E Z$, cells start to expand with rate $r_{\text {growthEZ}} / \mu \mathrm{m}$ until a maximum cell height of $175 \mu \mathrm{m}$ is reached and cells enter the $D Z$. MZ and EZ growth rates are per $\mu \mathrm{m}$, resulting in higher per cell growth rates for larger cells and constant elemental growth rates, consistent with experimental observations (Beemster and Baskin, 1998). Given the discrete, grid based nature of our model, cellular growth is executed in discrete steps during which a single row of grid points is added to the height of a cell. The time interval at which these discrete growth event occurs follows from the cellular growth rate in the following manner: if

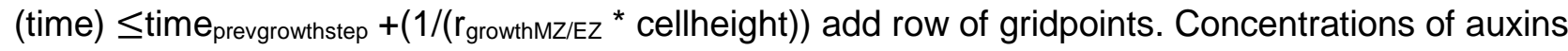
and proteins are corrected for these instantaneous cellular volume increases in case of cytoplasmic growth, but not in case of vacuolar driven cell expansion where cytoplasmic volume is assumed to stay constant. Upon division, cells are divided into two equally sized daughter cells that inherit transporter patterns and concentrations of cellular components of their mother cell. All tissues grow in the described manner. In the LRC developmental zones are shorter and cellular apoptosis occurs when cells reach a fixed position from the root tip, corresponding with the start of EZ of other tissue types (Fig.1A). To ensure an approximately constant size of the simulated tissue, a constant sized simulation domain encompassing the simulated tissue is defined and the most shootward cells are removed if their shootward cell wall is within 2 grid points of the simulation domain upper boundary.

\section{Model variations, auxin availability and transport}

To investigate the impact of root tip auxin transport and auxin availability, simulations with altered expression and/or localization of auxin importers and exporters or altered auxin production were performed (Fig 1C, 1D, 1E). Alterations in transporter levels, auxin production rates or shoot auxin influx rates were applied by simply multiplying default parameter values with a scaling parameter $\alpha$, using $\alpha>1$ in case of increase and $\alpha<1$ in case of decrease of transporter or production levels. Alterations were often applied in a tissue and zone specific manner, applying $a \neq 1$ only in specific regions of the root tip (Method Table 2). 
Method Table 2; Auxin availability and transport

\begin{tabular}{|l|l|l|l|}
\hline & Parameter & Affected tissue & $\alpha$ \\
\hline Low auxin production LRC (Fig 1C) & \multicolumn{1}{|l}{$L R C_{p_{A}}$} & LRC & 0.01 \\
\hline Low shoot influx (Fig 1C) & & - & 0.667 \\
\hline Low auxin production QC (Fig 1C) & \multicolumn{1}{|c|}{$Q C_{p_{A}}$} & QC & 0.01 \\
\hline Only downward PIN1 (Fig 1D) & PIN $_{\text {prepat }}$ & $\begin{array}{l}\text { All except vasculature and } \\
\text { pericycle }\end{array}$ & 0.0 \\
\hline Aux1 mutant (Fig 1D) & AUX/LAX $_{\text {pat }}$ & All & 0.1 \\
\hline Reduced PIN2 (Fig 1D) & PIN $_{\text {prepat }}$ & $\begin{array}{l}\text { EZ/DZ, LRC, epidermis and } \\
\text { cortex }\end{array}$ & 0.5 \\
\hline Extra AUX1 vasculature (Fig 1E) & AUX/LAX $_{\text {pat }}$ & Vasculature & 2.0 \\
\hline Extra AUX1 epidermis (Fig 1E) & AUX/LAX $_{\text {pat }}$ & Epidermis & 2.0 \\
\hline Only shootward PIN2(Fig 1E) & PIN $_{\text {prepat }}$ & $\begin{array}{l}\text { TZ/EZ/DZ, LRC, epidermis and } \\
\text { cortex (lateral membranes) }\end{array}$ & 0 \\
\hline
\end{tabular}

\section{Model variations, altered tissue specific growth dynamics}

For adjustment in growth dynamics for LRC tissue (Fig 2A) we assumed that the location of LRC shedding was more shootward to mimic a smb mutant while keeping all other settings the same. For simulations with absence of growth in specific tissue (Fig. 2A, 2B), simulations were run to equilibrium. After this, for assigned tissues no growth, division and expansion dynamics were simulated while all other tissue would continue growth as in default conditions.

\section{Model variations, auxin transport relative to cell height}

To determine the mechanism underlying the cell expansion driven increases in cellular auxin levels we performed additional simulations investigating the roles of auxin export and import and effective changes therein as a consequence of cell expansion. Since passive auxin uptake occurs across the membrane, which surface area increases with cell height passive transport automatically increases with cell height. This increase will thus also occur in planta. Additionally, for the apolar AUX/LAX-mediated active auxin import we did not incorporate a cell size increase mediated dilution of membrane transporter levels in our default model settings. Constant membrane levels with an increased membrane area result in larger cellular AUX/LAX levels, therefore, also active auxin import increases with cell height in our model. It is unclear whether in planta also such an increase occurs, which would imply an upregulation of AUX/LAX production proportionate to membrane area. Finally, PIN proteins typically are highly abundant on shootward/rootward membranes, with significantly lower levels and/or occupying smaller membrane fractions of lateral membranes. Nonetheless, analogous to the situation for AUX/LAX, the strong increase in lateral membrane length during growth results in an implicit upregulation of total lateral PIN levels elongation. Again it is unclear whether this increase occurs in planta, or rather total lateral PIN levels are maintained and smeared out over a larger area.

To investigate the relevance of these size-dependent increases in passive and active auxin import and export for auxin loading, in a subset of simulations we prevented this increase through normalizing these auxin fluxes for cell height in the TZ, EZ and DZ of stele cell files by multiplying them with a factor $\frac{M Z_{\text {cellheight }}}{\text { cellheight }}$ for cellheight $>2.2^{*} M Z_{\text {cellheight, }}$ where $M Z_{\text {cellheight }}(=8 \mu \mathrm{m})$ and 
$2^{*} M Z_{\text {cellheight }}(=16 \mu \mathrm{m})$ is the average cell height in the $\mathrm{TZ}$ just before expansion starts. Since plant cells elongate in length this normalization for transport was only applied for the lateral membranes. Furthermore, in case of normalization of passive import, since auxin levels in the EZ strongly depend on this passive influx we restricted the normalization to $25 \%$ of the total passive influx capacity to prevent a total auxin collapse in the simulations.

\section{Model variations, tissue specific zonation}

In our default simulations cell growth, division and expansion dynamics are perfectly synchronized within and across cell files. However, in planta between tissue types differences in zonation dynamics, cell cycle durations and cell sizes have been observed (Beemster and Baskin, 1998; Lavrekha et al., 2017; Rahni and Birnbaum, 2019). Stele protoxylem and protophloem cells start expanding relatively close to the root tip $(\sim 150 \mu \mathrm{m})$, whereas pericycle protoxylem and protophloem cells stop divisions furthest from the root tip $(\sim 250 \mu \mathrm{m})$ (Lavrekha et al., 2017; Rahni and Birnbaum, 2019). Additionally, vasculature cell cycles are faster than cell cycles in the outer tissues (16h vasculature versus $22 \mathrm{~h}$ cortex) ((Lavrekha et al., 2017), supplemental information) and vasculature cells have an increased height compared to outer tissue (1.5-2 times larger on average) (Lavrekha et al., 2017; Rahni and Birnbaum, 2019).

To investigate the consequences for priming of these cell type specific differences, we implemented a tissue type dependent location of the MZ-TZ boundary, transit amplifying division rates and cell sizes, both alone and in combination in our model. For the MZ-TZ boundary vasculature division ceases at $55 \%$ of the total meristem length, pericycle at $85 \%$ of meristem length and all other tissue at $75 \%$ of meristem length, for division rates we increased vascular TA division rates by a factor of 1.5 , and for cell sizes we increased vascular cell sizes by a factor of 1.5 .

Since plants cells have cell walls which they share with their neighboring cells, cells are unable to slide past one another and instead maintain their neighborhood of surrounding cells. This begs the question how vasculature cells can have a more rapid cell cycle, implying a larger doubling rate and hence elemental growth rate, compared to other cells. Adding to this a larger cell size (doubling in less time) aggravates this matter even further. However, while outer cell files curve out laterally from the QC (Fig 1A) and straighten out further shootward, vasculature cell files originate atop of the QC and follow a straight trajectory. We thus hypothesized that the curvature of nonvascular cell files would result in a longer pathlength, that in absence of compensation would result in a higher cumulative displacement at the end of the $\mathrm{MZ}$ for these cell files. This would imply that higher vascular growth rates serve to compensate for the longer pathlength. To investigate this matter, we analyzed 10 meristems of 7 -day old Arabidopsis roots grown on 1/2MS medium that were used for the lateral root number assay, We applied the Fuji plugin cell-o-tape to measure the length of the cell file from QC to EZ for a cortical and vasculature file and divided the difference of the cell file lengths through the average cortical cell size in the first 4 layers above the QC. The results indicate that a cortical cell file length is $12-20 \mu \mathrm{m}$ longer than stele file length, translating to roughly to $2.5-3$ cells of $5 \mu \mathrm{m}$. This indicates that $\sim 1-2$ division evens have occurred in the cortical cell file before vasculature divisions start.

We combined these findings with the data from Lavrehka 2017 et al regarding cell cycle and cell size values for cortex and stele cells to analytically test whether the differences in division rate and cell size indeed serve to compensate differences in cell file length and result in overall synchronized cumulative displacement from cell growth (Lavrekha et al., 2017).

For stele cells we model the cumulative displacement as function of time as:

$M Z_{\text {position }}(t)=2^{\frac{t}{t T A}}+2^{\frac{t-t S C}{t T A}}+2^{\frac{t-2 * t S C}{t T A}}$

Where $M Z_{\text {position }}$ is the position of a cell in a meristem expressed in cortical cell size, the terms are the growth contribution by TA division from released SC assuming that a maximum of 3 SC divisions occur in the time window that an individual cell spends in the MZ. When assuming a 1.5 times larger cell height. 
For the cortical cell files starting with 4 instead of 1 cell, we applied the following formula:

$M Z_{\text {position }}(t)=-3+4 * 2^{\frac{t}{t T A}}+2^{\frac{t-t S C}{t T A}}+2^{\frac{t-2 * t S C}{t T A}}$

Here the -3 term represents the 3 cells already present when counting from the QC in the cortical but not the vasculature cell file, to ensure we are comparing positions of cells with an initially corresponding position over time. Additionally, the -1.5tTAcort term represents that given the presence of 4 instead of 1 cells relative to the last SC division already 1 TA division has passed and hence a new SC division is due at time $\mathrm{t}=\mathrm{tSC}$-tTA. For stem cell cell cycles we applied $\mathrm{tSC}=60 \mathrm{~h}$ and for TA cell cycles we applied $\mathrm{tTA} \mathrm{A}_{\text {stele }}=16 \mathrm{~h}$ and $\mathrm{tTA} \mathrm{A}_{\text {cort }}=22 \mathrm{~h}$ for stele and cortex respectively (Lavrekha et al., 2017; Rahni and Birnbaum, 2019).

When plotting the above formulas while not incorporating the larger size of vascular cells nor their faster TA division (using the same value as for the cortex), we see that the head start provided by the curvature of the cortical cell file results in a substantially faster displacement of cortical versus vasculature cells (Methods Figure 3, yellow vs brown line). When incorporating in the vasculature formula either the larger cell size (Methods Figure 3, purple line) or the faster division rates this difference in displacement decreases (Methods Figure 3, grey line). Only when incorporating both aspects a highly similar displacement graph as compared to the cortex arises (Methods Figure 3, red line). These findings confirm our hypothesis, supporting that the larger vasculature cell size and faster division rates serve to compensate for the longer cell file length in the cortex due to curvature.

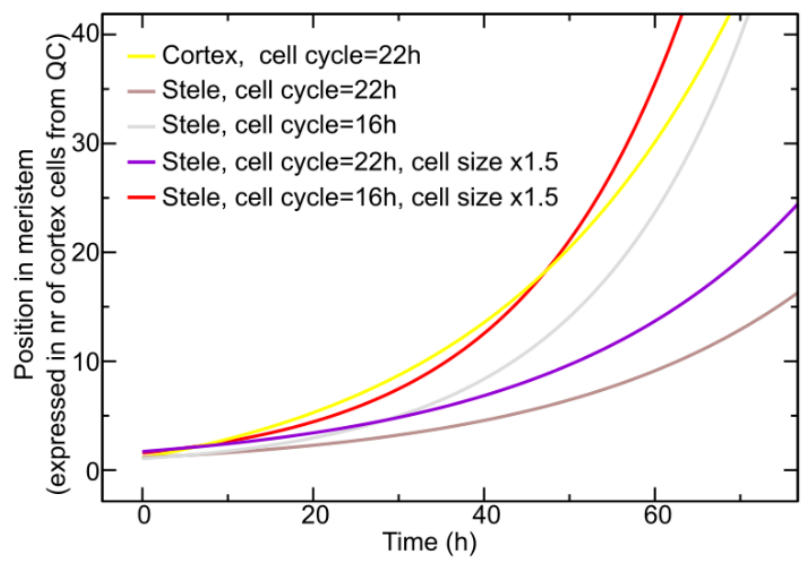

Methods Figure 3 Cumulative displacement of cortical and stele cell files with cortical cell cycle $=22 \mathrm{~h}$ and stele cell cycle $=16 \mathrm{~h}$ and cell size scaled such that cortical cell size $=1$ and stele cell size $=1.5$. Cortical cell displacement in meristem when applying cortex formula and cell cycles (yellow). Stele cell displacement applying stele formula combined with cortical cell cycle and cell size(brown), stele cell cycle and cortical cell size (grey), cortical cell cycle and stele cell size (purple) and stele cell cycle and cell size(red).

Since in our model growth dynamics are only applied outside of the curved region (see section Tissue layout), applying a faster division and hence growth rate in the vasculature would induce biologically unrealistic sliding in our model. To avoid this sliding, several adjustments to the model growth dynamics were made. First, under default conditions, per update step cells in the MZ could undergo either growth or division, leading to a small growth disadvantage of faster dividing cells. To avoid this, cells were allowed to both grow and divide during the same time step. Secondly in our default simulations cells enter the EZ when their lower membrane is above the LRC, however, with different cell sizes in the meristem this might result in large cells not meeting this condition yet while a smaller neighboring cell already enters the EZ and starts to rapidly elongate. To achieve across cell file synchronously occurring rapid elongation, cells should in addition to their lower membrane being above the LRC, have only neighboring cells in the horizontal plane that all also fulfill the requirements to start elongation. Finally, we applied a compensation in the elongation rates for large cells. To understand this, we need to consider the following: In our discrete, gridbased model, cell walls take up 1 grid point. Therefore, modeling the situation of a large cell 
flanked by two smaller cells half its size, results in our model in practice in a large cell of height $\mathrm{H}$ flanked by two cells of height $(\mathrm{H}-1) / 2$, due to the 1 grid point cell wall separating these two smaller cells also taking up space. As a consequence, despite having a constant elemental growth rate, the presence of a non-expanding cell wall causes the 2 small cells to expand less rapidly than the one larger cell. To prevent this, we need to downscale expansion rates of larger cells, taking into account the number of non-growing cell walls potentially present in cell files containing smaller cells.

To achieve this we apply the following formula:

$\mathrm{r}_{\text {growthMz/EZ }}$ * (cellheight-(cellheight/MZ $\left.\left.Z_{\text {cellheight }}-1\right)^{*} 1.5\right)$

giving a penalty for how often a meristematic cell size $\left(M Z_{\text {cellheight }}\right)$ fits in the cell size and hence compensating for the number of walls small $M Z_{\text {cellheight }}$ sized cells would have that do not contribute to growth rate, the -1 is such that a $M Z_{\text {cellheight }}$ sized cell has no penalty.

\section{Analysis methods (kymographs)}

To display and analyze simulated spatio-temporal auxin dynamics, we also generated model kymographs. Kymographs were created by taking snapshots of auxin patterns in a one grid point wide longitudinal cross section in the cell file of interest (typically outer vasculature or pericycle). To zoom in on the spatial domain relevant for priming, longitudinal snapshots run from the first dividing cell $(\sim 200 \mu \mathrm{m}$ from root tip) to the position where cells are fully elongated $(\sim 1200 \mu \mathrm{m}$ from the root tip). Snapshots were stored every 100 time steps (=20 seconds) and aligned according to their temporal sequence. Priming frequency was obtained by $\frac{\text { priming-1 }}{\text { firstime }}$, where priming is the observed number of priming events, time is the simulation time. A cell was considered to undergo priming (i.e. its passing through the EZ was counted as a priming event) when the auxin level of that cell at the start of the EZ was more than $110 \%$ of that of it's immediate above and below neighboring cells Priming site spacing was counted as the number of cells passing through in the time interval between 2 priming events, cells undergoing priming were excluded from the priming site spacing count.

\section{Numerical integration and run-time performance}

Auxin transport occurs at relatively high rates. As a consequence, standard Euler forward explicit integration schemes would require very small temporal integration steps $(\Delta t=0.0001)$. To simulate plant growth dynamics over a time course of one or several days, this would result in excessively long simulation run times. Therefore, similar to earlier modeling studies by us and others (Grieneisen et al., 2007; Mahonen et al., 2014) we used an alternating direction semi-implicit integration scheme for the auxin partial differential equations (Peaceman and Rachford, 1955), allowing us to use integration steps of $0.2 \mathrm{~s}$ and a spatial integration step of $\Delta x=2 \mu \mathrm{m}$. The code of the model was written in $\mathrm{C}_{++}$, simulations were run on 24 to 36 -core workstations with Intel Xeon E5-2687W processors, resulting in a typical run-time of 24 hours for a simulation representing 6 days of plant growth.

\section{QUANTIFICATION AND STATISTICAL ANALYSIS}

Statistical analysis and curve fitting were performed using python. Criteria for exclusion of experimental samples were described in the corresponding STAR methods. Statistical details of experiments (whenever present: $n$, mean, median, $p$-value) are in the figure legends of corresponding experiments.

\section{Computational results}

\section{Robustness analysis}

To test whether the observed behavior found here might be an artifact of modelling choices we performed an extensive robustness analysis. First, to ensure that the observed oscillations are not a result of changes in tissue total auxin content due to the culling of the most shootward cells as they are nearing the boundary of the simulation domain, we performed simulations in much larger 
simulations domains yet similar starting tissue sizes in which initially the simulation domain is not reached by the topmost cells (Supp. 3: Fig. 1). This enabled us to validate that oscillations occur also in absence of culling of shootward cells. Second, to ensure robustness against changes in precise root tip architecture or division dynamics incorporated in the model, simulations were performed in an alternative root tip architecture with a more pointy root tip shape as previously used in our halotropism study (van den Berg et al., 2016) (Supp. 3: Fig. 2), in a root tip architecture in which all cells have a reduced width (Supp. 3: Fig. 3), in a root tip layout in which cells in the different cell files are staggered (Supp. 3: Fig. 4), in a root tip in which clones of sibling cells divide slightly asynchronously (Supp. 3: Fig. 5), and in a root tip where both different cell files are staggered and cells divide slightly asynchronous (Supp. 3: Fig. 6). In all cases, oscillation dynamics were found to robustly occur. Additionally, simulations were performed at an alternative spatial resolution $(2 \mu \mathrm{m}$ instead of the default $1 \mu \mathrm{m})$, again without resulting in changes in oscillation dynamics (Supp. 3: Fig. 3). Next, we investigated whether the incorporated auxindependence of AUX/LAX expression, or rather ignoring the auxin-dependence of PIN expression affected oscillations. Again, both removing AUX/LAX auxin dependence or instead incorporating PIN auxin dependence had no significant effect on oscillation dynamics (Supp. 3: Fig. 7, Fig. 8). Finally, we performed simulations for a root tip model incorporating multiple layers of cortical cell layers as frequently encountered in plant species other than Arabidopsis thaliana demonstrating that also this does not influence priming dynamics (Supp. 3: Fig. 9). We thus conclude that the observed oscillations in auxin levels are not a direct result of particular choices in model assumptions, simplifications or implementation.

In the main manuscript, as part of our investigation of the mechanism underlying oscillations we have varied auxin production rates and locations, PIN and AUX1 transporter efficiencies and patterns, meristem sizes, and cell division and elongation rates. In all cases, periodic auxin oscillations were observed. Changes in auxin parameters merely affected oscillation amplitude but not frequency, as for example shown in Figure 1C and 1D. Similarly, changes in elongation rate also affected oscillation amplitude but not frequency, as demonstrated in Figure 4. In contrast, changes in meristem size and division rate affected priming frequency and spacing, as described. Only when the auxin reflux loop was fully abolished (Fig 1E, yellow line) or vascular cells were not allowed to grow (Fig S2B) simulations did not display periodic auxin oscillations. 


\section{References:}

Alarcon, M.V., Salguero, J., and Lloret, P.G. (2019). Auxin Modulated Initiation of Lateral Roots Is Linked to Pericycle Cell Length in Maize. Front Plant Sci 10, 11.

Baker, R.E., Schnell, S., and Maini, P.K. (2006). A clock and wavefront mechanism for somite formation. Dev Biol 293, 116-126.

Band, L.R., Wells, D.M., Fozard, J.A., Ghetiu, T., French, A.P., Pound, M.P., Wilson, M.H., Yu, L., Li, W., Hijazi, H.I., et al. (2014). Systems Analysis of Auxin Transport in the Arabidopsis Root Apex. The Plant Cell 26, 862-875.

Bao, Y., Aggarwal, P., Robbins, N.E., 2nd, Sturrock, C.J., Thompson, M.C., Tan, H.Q., Tham, C., Duan, L., Rodriguez, P.L., Vernoux, T., et al. (2014). Plant roots use a patterning mechanism to position lateral root branches toward available water. Proc Natl Acad Sci U S A 111, 9319-9324. Beemster, G.T., and Baskin, T.I. (1998). Analysis of cell division and elongation underlying the developmental acceleration of root growth in Arabidopsis thaliana. Plant Physiol 116, 1515-1526. Bennett, M.J., Marchant, A., Green, H.G., May, S.T., Ward, S.P., Millner, P.A., Walker, A.R., Schulz, B., and Feldmann, K.A. (1996). Arabidopsis AUX1 gene: a permease-like regulator of root gravitropism. Science 273, 948-950.

Bessho, Y., Hirata, H., Masamizu, Y., and Kageyama, R. (2003). Periodic repression by the bHLH factor Hes7 is an essential mechanism for the somite segmentation clock. Genes Dev 17, 14511456.

Bhalerao, R.P., Eklof, J., Ljung, K., Marchant, A., Bennett, M., and Sandberg, G. (2002). Shootderived auxin is essential for early lateral root emergence in Arabidopsis seedlings. Plant J 29, 325332.

Bielach, A., Podlesakova, K., Marhavy, P., Duclercq, J., Cuesta, C., Muller, B., Grunewald, W., Tarkowski, P., and Benkova, E. (2012). Spatiotemporal regulation of lateral root organogenesis in Arabidopsis by cytokinin. Plant Cell 24, 3967-3981.

Bizet, F., Hummel, I., and Bogeat-Triboulot, M.B. (2015). Length and activity of the root apical meristem revealed in vivo by infrared imaging. J Exp Bot 66, 1387-1395.

Campilho, A., Garcia, B., Toorn, H.V., Wijk, H.V., Campilho, A., and Scheres, B. (2006). Timelapse analysis of stem-cell divisions in the Arabidopsis thaliana root meristem. Plant J 48, 619-627. Conlon, R.A., Reaume, A.G., and Rossant, J. (1995). Notch1 is required for the coordinate segmentation of somites. Development 121, 1533-1545.

Cooke, J., and Zeeman, E.C. (1976). A clock and wavefront model for control of the number of repeated structures during animal morphogenesis. J Theor Biol 58, 455-476.

Cruz-Ramirez, A., Diaz-Trivino, S., Blilou, I., Grieneisen, V.A., Sozzani, R., Zamioudis, C., Miskolczi, P., Nieuwland, J., Benjamins, R., Dhonukshe, P., et al. (2012). A bistable circuit involving SCARECROW-RETINOBLASTOMA integrates cues to inform asymmetric stem cell division. Cell 150, 1002-1015.

Davis, G.K., and Patel, N.H. (1999). The origin and evolution of segmentation. Trends in Cell Biology 9, M68-M72.

De Rybel, B., Audenaert, D., Xuan, W., Overvoorde, P., Strader, L.C., Kepinski, S., Hoye, R., Brisbois, R., Parizot, B., Vanneste, S., et al. (2012). A role for the root cap in root branching revealed by the non-auxin probe naxillin. Nat Chem Biol 8, 798-805.

De Smet, I., Tetsumura, T., De Rybel, B., Frei dit Frey, N., Laplaze, L., Casimiro, I., Swarup, R., Naudts, M., Vanneste, S., Audenaert, D., et al. (2007). Auxin-dependent regulation of lateral root positioning in the basal meristem of Arabidopsis. Development 134, 681-690.

Dello Ioio, R., Nakamura, K., Moubayidin, L., Perilli, S., Taniguchi, M., Morita, M.T., Aoyama, T., Costantino, P., and Sabatini, S. (2008). A genetic framework for the control of cell division and differentiation in the root meristem. Science 322, 1380-1384. 
Dequeant, M.L., Glynn, E., Gaudenz, K., Wahl, M., Chen, J., Mushegian, A., and Pourquie, O. (2006). A complex oscillating network of signaling genes underlies the mouse segmentation clock. Science 314, 1595-1598.

Di Mambro, R., De Ruvo, M., Pacifici, E., Salvi, E., Sozzani, R., Benfey, P.N., Busch, W., Novak, O., Ljung, K., Di Paola, L., et al. (2017). Auxin minimum triggers the developmental switch from cell division to cell differentiation in the Arabidopsis root. Proc Natl Acad Sci U S A 114, E7641E7649.

DiDonato, R.J., Arbuckle, E., Buker, S., Sheets, J., Tobar, J., Totong, R., Grisafi, P., Fink, G.R., and Celenza, J.L. (2004). Arabidopsis ALF4 encodes a nuclear-localized protein required for lateral root formation. Plant J 37, 340-353.

Dolle, P., Izpisua-Belmonte, J.C., Falkenstein, H., Renucci, A., and Duboule, D. (1989). Coordinate expression of the murine Hox-5 complex homoeobox-containing genes during limb pattern formation. Nature 342, 767-772.

Du, Y., and Scheres, B. (2017). PLETHORA transcription factors orchestrate de novo organ patterning during Arabidopsis lateral root outgrowth. Proc Natl Acad Sci U S A 114, 11709-11714. Dubrovsky, J.G., Gambetta, G.A., Hernandez-Barrera, A., Shishkova, S., and Gonzalez, I. (2006). Lateral root initiation in Arabidopsis: developmental window, spatial patterning, density and predictability. Ann Bot 97, 903-915.

Dubrovsky, J.G., Sauer, M., Napsucialy-Mendivil, S., Ivanchenko, M.G., Friml, J., Shishkova, S., Celenza, J., and Benkova, E. (2008). Auxin acts as a local morphogenetic trigger to specify lateral root founder cells. Proc Natl Acad Sci U S A 105, 8790-8794.

Dubrulle, J., McGrew, M.J., and Pourquie, O. (2001). FGF signaling controls somite boundary position and regulates segmentation clock control of spatiotemporal Hox gene activation. Cell 106, 219-232.

Dubrulle, J., and Pourquié, O. (2002). From head to tail: links between the segmentation clock and antero-posterior patterning of the embryo. Current Opinion in Genetics \& Development 12, 519523.

el-Showk, S., Help-Rinta-Rahko, H., Blomster, T., Siligato, R., Maree, A.F., Mahonen, A.P., and Grieneisen, V.A. (2015). Parsimonious Model of Vascular Patterning Links Transverse Hormone Fluxes to Lateral Root Initiation: Auxin Leads the Way, while Cytokinin Levels Out. PLoS Comput Biol 11, e1004450.

Eshel, A., and Beeckman, T. (2013). Plant Roots.

French, A.P., Wilson, M.H., Kenobi, K., Dietrich, D., Voss, U., Ubeda-Tomas, S., Pridmore, T.P., and Wells, D.M. (2012). Identifying biological landmarks using a novel cell measuring image analysis tool: Cell-o-Tape. Plant Methods 8, 7.

Galway, M.E., Masucci, J.D., Lloyd, A.M., Walbot, V., Davis, R.W., and Schiefelbein, J.W. (1994). The TTG gene is required to specify epidermal cell fate and cell patterning in the Arabidopsis root. Dev Biol 166, 740-754.

Gonzalez-Garcia, M.P., Vilarrasa-Blasi, J., Zhiponova, M., Divol, F., Mora-Garcia, S., Russinova, E., and Cano-Delgado, A.I. (2011). Brassinosteroids control meristem size by promoting cell cycle progression in Arabidopsis roots. Development 138, 849-859.

Grieneisen, V.A., Xu, J., Marée, A.F., Hogeweg, P., and Scheres, B. (2007). Auxin transport is sufficient to generate a maximum and gradient guiding root growth. Nature 449, 1008-1013.

Gruber, B.D., Giehl, R.F., Friedel, S., and von Wiren, N. (2013). Plasticity of the Arabidopsis root system under nutrient deficiencies. Plant Physiol 163, 161-179.

Hayashi, K., Hasegawa, J., and Matsunaga, S. (2013). The boundary of the meristematic and elongation zones in roots: endoreduplication precedes rapid cell expansion. Sci Rep 3, 2723. Hofhuis, H., Laskowski, M., Du, Y., Prasad, K., Grigg, S., Pinon, V., and Scheres, B. (2013). Phyllotaxis and rhizotaxis in Arabidopsis are modified by three PLETHORA transcription factors. Curr Biol 23, 956-962.

Ishida, T., Kurata, T., Okada, K., and Wada, T. (2008). A genetic regulatory network in the development of trichomes and root hairs. Annu Rev Plant Biol 59, 365-386. 
Ivanov, V.B., and Dubrovsky, J.G. (2013). Longitudinal zonation pattern in plant roots: conflicts and solutions. Trends Plant Sci 18, 237-243.

Jansen, L., Roberts, I., De Rycke, R., and Beeckman, T. (2012). Phloem-associated auxin response maxima determine radial positioning of lateral roots in maize. Philos Trans R Soc Lond B Biol Sci $367,1525-1533$.

Jensen, P.J., Hangarter, R.P., and Estelle, M. (1998). Auxin transport is required for hypocotyl elongation in light-grown but not dark-grown Arabidopsis. Plant Physiol 116, 455-462.

Jiang, T.X., Jung, H.S., Widelitz, R.B., and Chuong, C.M. (1999). Self-organization of periodic patterns by dissociated feather mesenchymal cells and the regulation of size, number and spacing of primordia. Development 126, 4997-5009.

Jiang, Y.J., Aerne, B.L., Smithers, L., Haddon, C., Ish-Horowicz, D., and Lewis, J. (2000). Notch signalling and the synchronization of the somite segmentation clock. Nature 408, 475-479.

John C. Larkin, Jason D. Walker, Agnese C. Bolognesi-Winfield, Gray, J.C., and Walker, A.R. (1999). Allele-Specific Interactions Between ttg and gl1 During Trichome Development in Arabidopsis thaliana. Genetics 151, 1591-1604.

Jonsson, H., Heisler, M.G., Shapiro, B.E., Meyerowitz, E.M., and Mjolsness, E. (2006). An auxindriven polarized transport model for phyllotaxis. Proc Natl Acad Sci U S A 103, 1633-1638.

Kerstens, M., Strauss, S., Smith, R., and Willemsen, V. (2020). From Stained Plant Tissues to Quantitative Cell Segmentation Analysis with MorphoGraphX. Methods Mol Biol 2122, 63-83. Kircher, S., and Schopfer, P. (2016). Priming and positioning of lateral roots in Arabidopsis. An approach for an integrating concept. J Exp Bot 67, 1411-1420.

Kircher, S., and Schopfer, P. (2018). The plant hormone auxin beats the time for oscillating lightregulated lateral root induction. Development 145.

Laskowski, M. (2013). Lateral root initiation is a probabilistic event whose frequency is set by fluctuating levels of auxin response. J Exp Bot 64, 2609-2617.

Laskowski, M., Biller, S., Stanley, K., Kajstura, T., and Prusty, R. (2006). Expression profiling of auxin-treated Arabidopsis roots: toward a molecular analysis of lateral root emergence. Plant Cell Physiol 47, 788-792.

Laskowski, M., Grieneisen, V.A., Hofhuis, H., Hove, C.A., Hogeweg, P., Maree, A.F., and Scheres, B. (2008). Root system architecture from coupling cell shape to auxin transport. PLoS Biol 6, e307. Laskowski, M., and Ten Tusscher, K.H. (2017). Periodic Lateral Root Priming: What Makes It Tick? Plant Cell 29, 432-444.

Lavrekha, V.V., Pasternak, T., Ivanov, V.B., Palme, K., and Mironova, V.V. (2017). 3D analysis of mitosis distribution highlights the longitudinal zonation and diarch symmetry in proliferation activity of the Arabidopsis thaliana root meristem. Plant J 92, 834-845.

Lewis, D.R., Negi, S., Sukumar, P., and Muday, G.K. (2011). Ethylene inhibits lateral root development, increases IAA transport and expression of PIN3 and PIN7 auxin efflux carriers. Development 138, 3485-3495.

Mahonen, A.P., Ten Tusscher, K., Siligato, R., Smetana, O., Diaz-Trivino, S., Salojarvi, J., Wachsman, G., Prasad, K., Heidstra, R., and Scheres, B. (2014). PLETHORA gradient formation mechanism separates auxin responses. Nature 515, 125-129.

McGinnis, W., and Krumlauf, R. (1992). Homeobox genes and axial patterning. Cell 68, 283-302. Moreno-Risueno, M.A., Van Norman, J.M., Moreno, A., Zhang, J., Ahnert, S.E., and Benfey, P.N. (2010). Oscillating gene expression determines competence for periodic Arabidopsis root branching. Science 329, 1306-1311.

Newman, S.A., and Frisch, H.L. (1979). Dynamics of skeletal pattern formation in developing chick limb. Science 205, 662-668.

Nieuwland, J., Maughan, S., Dewitte, W., Scofield, S., Sanz, L., and Murray, J.A. (2009). The Dtype cyclin CYCD4;1 modulates lateral root density in Arabidopsis by affecting the basal meristem region. Proc Natl Acad Sci U S A 106, 22528-22533. 
Novak, D., Kucharova, A., Ovecka, M., Komis, G., and Samaj, J. (2016). Developmental Nuclear Localization and Quantification of GFP-Tagged EB1c in Arabidopsis Root Using Light-Sheet Microscopy. Front Plant Sci 6, 1187.

Omelyanchuk, N.A., Kovrizhnykh, V.V., Oshchepkova, E.A., Pasternak, T., Palme, K., and Mironova, V.V. (2016). A detailed expression map of the PIN1 auxin transporter in Arabidopsis thaliana root. BMC Plant Biol 16 Suppl 1, 5.

Orosa-Puente, B., Leftley, N., von Wangenheim, D., Banda, J., Srivastava, A.K., Hill, K., Truskina, J., Bhosale, R., Morris, E., Srivastava, M., et al. (2018). Root branching toward water involves posttranslational modification of transcription factor ARF7. Science 362, 1407-1410.

Palmeirim, I., Henrique, D., Ish-Horowicz, D., and Pourquie, O. (1997). Avian hairy gene expression identifies a molecular clock linked to vertebrate segmentation and somitogenesis. Cell 91, 639-648.

Panganiban, G., Irvine, S.M., Lowe, C., Roehl, H., Corley, L.S., Sherbon, B., Grenier, J.K., Fallon, J.F., Kimble, J., Walker, M., et al. (1997). The origin and evolution of animal appendages. Proc Natl Acad Sci U S A 94, 5162-5166.

Peaceman, D.W., and Rachford, J., Henry H (1955). The numerical solution of parabolic and elliptic differential equations. Journal of the Society for industrial and Applied Mathematics 3, 2841.

Péret, B., Swarup, K., Ferguson, A., Seth, M., Yang, Y., Dhondt, S., James, N., Casimiro, I., Perry, P., and Syed, A. (2012). AUX/LAX genes encode a family of auxin influx transporters that perform distinct functions during Arabidopsis development. The Plant Cell 24, 2874-2885.

Pérez-Torres, C.-A., López-Bucio, J., Cruz-Ramírez, A., Ibarra-Laclette, E., Dharmasiri, S., Estelle, M., and Herrera-Estrella, L. (2008). Phosphate Availability Alters Lateral Root Development in Arabidopsis by Modulating Auxin Sensitivity via a Mechanism Involving the TIR1 Auxin Receptor. The Plant Cell 20, 3258-3272.

Perianez-Rodriguez, J., Rodriguez, M., Marconi, M., Bustillo-Avendaño, E., Wachsman, G., Sanchez-Corrionero, A., De Gernier, H., Cabrera, J., Perez-Garcia, P., and Gude, I. (2021). An auxin-regulable oscillatory circuit drives the root clock in Arabidopsis. Science Advances 7, eabd4722.

Plikus, M.V., Mayer, J.A., de la Cruz, D., Baker, R.E., Maini, P.K., Maxson, R., and Chuong, C.M. (2008). Cyclic dermal BMP signalling regulates stem cell activation during hair regeneration. Nature 451, 340-344.

Rahni, R., and Birnbaum, K.D. (2019). Week-long imaging of cell divisions in the Arabidopsis root meristem. Plant Methods 15, 30.

Raspopovic, J., Marcon, L., Russo, L., and Sharpe, J. (2014). Modeling digits. Digit patterning is controlled by a Bmp-Sox9-Wnt Turing network modulated by morphogen gradients. Science 345 , 566-570.

Reinhardt, D., Pesce, E.R., Stieger, P., Mandel, T., Baltensperger, K., Bennett, M., Traas, J., Friml, J., and Kuhlemeier, C. (2003). Regulation of phyllotaxis by polar auxin transport. Nature 426, 255260.

Richter, G.L., Monshausen, G.B., Krol, A., and Gilroy, S. (2009). Mechanical stimuli modulate lateral root organogenesis. Plant Physiol 151, 1855-1866.

Rogers, E.D., and Benfey, P.N. (2015). Regulation of plant root system architecture: implications for crop advancement. Curr Opin Biotechnol 32, 93-98.

Salvi, E., Rutten, J.P., Di Mambro, R., Polverari, L., Licursi, V., Negri, R., Dello Ioio, R., Sabatini, S., and Ten Tusscher, K. (2020). A Self-Organized PLT/Auxin/ARR-B Network Controls the Dynamics of Root Zonation Development in Arabidopsis thaliana. Dev Cell 53, 431-443 e423. Sarrazin, A.F., Peel, A.D., and Averof, M. (2012). A segmentation clock with two-segment periodicity in insects. Science 336, 338-341.

Saunders, J.W., Jr. (1948). The proximo-distal sequence of origin of the parts of the chick wing and the role of the ectoderm. J Exp Zool 108, 363-403.

Saunders, P.T. (1992). Collected Works of A.M. Turing: Morphogenesis (Amsterdam: Elsevier). 
Schindelin, J., Arganda-Carreras, I., Frise, E., Kaynig, V., Longair, M., Pietzsch, T., Preibisch, S., Rueden, C., Saalfeld, S., Schmid, B., et al. (2012). Fiji: an open-source platform for biologicalimage analysis. Nat Methods 9, 676-682.

Shi, C.L., von Wangenheim, D., Herrmann, U., Wildhagen, M., Kulik, I., Kopf, A., Ishida, T., Olsson, V., Anker, M.K., Albert, M., et al. (2018). The dynamics of root cap sloughing in Arabidopsis is regulated by peptide signalling. Nat Plants 4, 596-604.

Shyer, A.E., Rodrigues, A.R., Schroeder, G.G., Kassianidou, E., Kumar, S., and Harland, R.M. (2017). Emergent cellular self-organization and mechanosensation initiate follicle pattern in the avian skin. Science 357, 811-815.

Sick, S., Reinker, S., Timmer, J., and Schlake, T. (2006). WNT and DKK determine hair follicle spacing through a reaction-diffusion mechanism. Science 314, 1447-1450.

Smith, R.S., Guyomarc'h, S., Mandel, T., Reinhardt, D., Kuhlemeier, C., and Prusinkiewicz, P. (2006). A plausible model of phyllotaxis. Proc Natl Acad Sci U S A 103, 1301-1306.

Strader, L.C., and Bartel, B. (2011). Transport and metabolism of the endogenous auxin precursor indole-3-butyric acid. Mol Plant 4, 477-486.

Swarup, K., Benkova, E., Swarup, R., Casimiro, I., Peret, B., Yang, Y., Parry, G., Nielsen, E., De Smet, I., Vanneste, S., et al. (2008). The auxin influx carrier LAX3 promotes lateral root emergence. Nat Cell Biol 10, 946-954.

Swarup, R., Friml, J., Marchant, A., Ljung, K., Sandberg, G., Palme, K., and Bennett, M. (2001). Localization of the auxin permease AUX1 suggests two functionally distinct hormone transport pathways operate in the Arabidopsis root apex. Genes \& development 15, 2648-2653.

Swarup, R., Kramer, E.M., Perry, P., Knox, K., Leyser, H.O., Haseloff, J., Beemster, G.T., Bhalerao, R., and Bennett, M.J. (2005). Root gravitropism requires lateral root cap and epidermal cells for transport and response to a mobile auxin signal. Nature cell biology 7, 1057-1065.

Toyokura, K., Goh, T., Shinohara, H., Shinoda, A., Kondo, Y., Okamoto, Y., Uehara, T., Fujimoto, K., Okushima, Y., Ikeyama, Y., et al. (2019). Lateral Inhibition by a Peptide Hormone-Receptor Cascade during Arabidopsis Lateral Root Founder Cell Formation. Dev Cell 48, 64-75 e65.

Turing, A.M. (1952). The chemical basis of morphogenesis. Philosophical Transactions of the Royal Society of London Series B, Biological Sciences 237, 37-72.

Ubeda-Tomas, S., Swarup, R., Coates, J., Swarup, K., Laplaze, L., Beemster, G.T., Hedden, P., Bhalerao, R., and Bennett, M.J. (2008). Root growth in Arabidopsis requires gibberellin/DELLA signalling in the endodermis. Nat Cell Biol 10, 625-628.

van den Berg, T., Korver, R.A., Testerink, C., and Ten Tusscher, K.H. (2016). Modeling halotropism: a key role for root tip architecture and reflux loop remodeling in redistributing auxin. Development 143, 3350-3362.

von Wangenheim, D., Hauschild, R., Fendrych, M., Barone, V., Benkova, E., and Friml, J. (2017). Live tracking of moving samples in confocal microscopy for vertically grown roots. Elife 6 .

Wachsman, G., Zhang, J., Moreno-Risueno, M.A., Anderson, C.T., and Benfey, P.N. (2020). Cell wall remodeling and vesicle trafficking mediate the root clock in Arabidopsis. Science 370, 819823.

Wendrich, J.R., Moller, B.K., Li, S., Saiga, S., Sozzani, R., Benfey, P.N., De Rybel, B., and Weijers, D. (2017). Framework for gradual progression of cell ontogeny in the Arabidopsis root meristem. Proc Natl Acad Sci U S A 114, E8922-E8929.

Willemsen, V., Wolkenfelt, H., De Vrieze, G., Weisbeek, P., and Scheres, B. (1998). The HOBBIT gene is required for formation of the root meristem in the Arabidopsis embryo. Development 125, 521-531.

Xiao, T.T., van Velzen, R., Kulikova, O., Franken, C., and Bisseling, T. (2019). Lateral root formation involving cell division in both pericycle, cortex and endodermis is a common and ancestral trait in seed plants. Development 146.

Xuan, W., Audenaert, D., Parizot, B., Moller, B.K., Njo, M.F., De Rybel, B., De Rop, G., Van Isterdael, G., Mahonen, A.P., Vanneste, S., et al. (2015). Root Cap-Derived Auxin Pre-patterns the Longitudinal Axis of the Arabidopsis Root. Curr Biol 25, 1381-1388. 
Xuan, W., Band, L.R., Kumpf, R.P., Van Damme, D., Parizot, B., De Rop, G., Opdenacker, D., Moller, B.K., Skorzinski, N., Njo, M.F., et al. (2016). Cyclic programmed cell death stimulates hormone signaling and root development in Arabidopsis. Science 351, 384-387.

Xuan, W., Opdenacker, D., Vanneste, S., and Beeckman, T. (2018). Long-Term In Vivo Imaging of Luciferase-Based Reporter Gene Expression in Arabidopsis Roots. Methods Mol Biol 1761, 177190.

Yamaguchi, M., Yoshimoto, E., and Kondo, S. (2007). Pattern regulation in the stripe of zebrafish suggests an underlying dynamic and autonomous mechanism. Proc Natl Acad Sci U S A 104, 47904793. 